\title{
Optimal Sequence Memory in Driven Random Networks
}

\author{
Jannis Schuecker, ${ }^{1,2}$ Sven Goedeke, ${ }^{1,3, *}$ and Moritz Helias ${ }^{1,4}$ \\ ${ }^{1}$ Institute of Neuroscience and Medicine (INM-6) and Institute for Advanced Simulation (IAS-6) \\ and JARA BRAIN Institute I, Jülich Research Centre, 52428 Jülich, Germany \\ ${ }^{2}$ Fraunhofer Center for Machine Learning and Fraunhofer IAIS, 53757 Sankt Augustin, Germany \\ ${ }^{3}$ Neural Network Dynamics and Computation, Institute of Genetics, University of Bonn, \\ 53115 Bonn, Germany \\ ${ }^{4}$ Department of Physics, Faculty 1, RWTH Aachen University, 52074 Aachen, Germany
}

(Received 28 September 2017; revised manuscript received 13 August 2018; published 14 November 2018)

\begin{abstract}
Autonomous, randomly coupled, neural networks display a transition to chaos at a critical coupling strength. Here, we investigate the effect of a time-varying input on the onset of chaos and the resulting consequences for information processing. Dynamic mean-field theory yields the statistics of the activity, the maximum Lyapunov exponent, and the memory capacity of the network. We find an exact condition that determines the transition from stable to chaotic dynamics and the sequential memory capacity in closed form. The input suppresses chaos by a dynamic mechanism, shifting the transition to significantly larger coupling strengths than predicted by local stability analysis. Beyond linear stability, a regime of coexistent locally expansive but nonchaotic dynamics emerges that optimizes the capacity of the network to store sequential input.
\end{abstract}

DOI: 10.1103/PhysRevX.8.041029

Subject Areas: Biological Physics, Interdisciplinary Physics,

Nonlinear Dynamics

\section{INTRODUCTION}

Large random networks of neuronlike units can exhibit collective chaotic dynamics [1-4]. Their information processing capabilities have been a focus in neuroscience [5] and in machine learning [6], and they show optimal performance close to the transition to chaos [7-9]. Because of its rich chaotic dynamics, the seminal network model by Sompolinsky et al. [1] serves as a model for various activity patterns observed in working memory tasks [10-13], motor control [14], and perceptual decision making [15]. The interplay between a time-dependent input signal and the dynamical state of the network, however, is poorly understood, notwithstanding consequences for information processing.

In the absence of a signal, the network dynamics is autonomous. Networks of randomly coupled rate neurons display a transition from a fixed point to chaotic fluctuations at a critical coupling strength [1], illustrated in Fig. 1(a). The transition is well understood by dynamic mean-field theory, originally developed for spin glasses $[16,17]$. The onset of chaos is equivalent to the emergence

*J.S. and S. G. contributed equally to this work.

Published by the American Physical Society under the terms of the Creative Commons Attribution 4.0 International license. Further distribution of this work must maintain attribution to the author(s) and the published article's title, journal citation, and DOI. of a nonzero, decaying autocorrelation function, whose decay time diverges at the transition. This equivalence has been used in several subsequent studies [18-20]. Furthermore, for autonomous systems, a tight relationship exists to random matrix theory $[21,22]$ : The transition from a fixed point to the chaotic state happens precisely when the fixed point becomes linearly unstable [1], which identifies the spectral radius of the random connectivity matrix as the parameter controlling the transition in such systems.

In stochastically driven systems, a direct relation between a decaying autocorrelation function, chaotic dynamics, and linear stability does not exist: Stochastic drive per se decorrelates the network activity even if the dynamics is stable [Fig. 1(b)], so a decaying autocorrelation function does not necessarily indicate chaos. The stochastic drive, furthermore, causes perpetual fluctuations in the regular regime as well. Therefore, a transition to chaos, if it exists at all, must be of a qualitatively different kind than the transition from the silent fixed point in the autonomous case. Time-dependent driving has indeed been found to stabilize network dynamics [18,23]. However, the mechanism is only understood for low-dimensional systems in the context of chaos synchronization by noise [24], in networks driven by deterministic signals [18], and in systems with discrete-time dynamics [23]. In the latter model, the effect of the stochastic input on the transition to chaos is completely captured by its influence on the spectral radius of the Jacobian matrix. Its single neuron dynamics, moreover, does not possess nontrivial temporal correlations. But these 
(a)
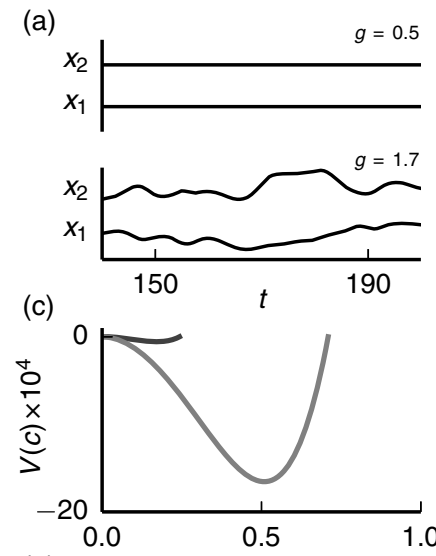

(e)

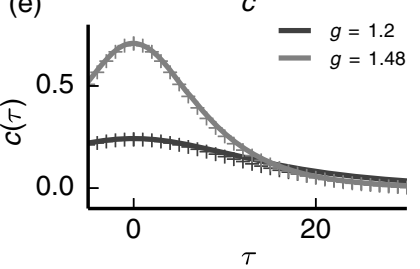

(f) (b)

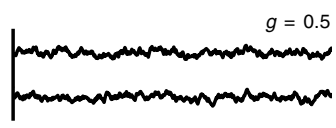

(d)
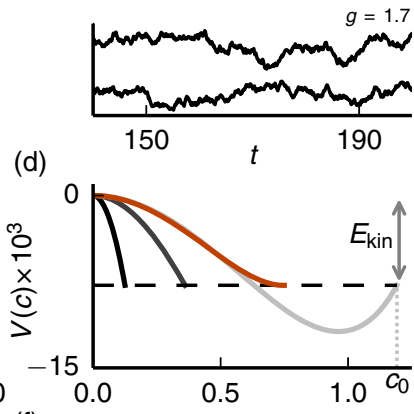

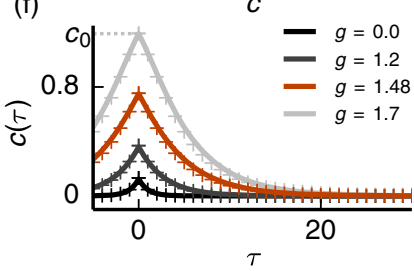

FIG. 1. Activity statistics of the autonomous and driven networks. The autonomous case is for $\sigma=0$ (left column), and the driven case is for $\sigma=0.35$ (right column). Upper row: Simulated trajectories of two example neurons for subcritical $g=0.5$ (upper part of vertical axis) and supercritical coupling $g=1.7$ (lower part of vertical axis). Middle row: Classical potential (10) with self-consistently determined variance $c_{0}$ following from energy conservation (11) for different coupling strengths $g$ (corresponding legends in lower row). The dashed horizontal line indicates minus initial kinetic energy $E_{\mathrm{kin}}=\sigma^{4} / 2$. In the driven case, the critical coupling $g_{c}=1.48$ from Eq. (20) is shown in red. Lower row: Self-consistent autocorrelation function (solid curve) compared to simulations (crosses). The variance (peak height) $c_{0}$ corresponds to the largest value of $c$ at which the potential (middle row) is defined, indicated for $g=1.7$ with gray dotted lines in (d) and (f). The network size in the simulations is $N=10000$.

temporal correlations are indeed essential for the transition to chaos and for information processing in continuous-time systems, as we show here.

Realistic continuous-time network models can generate complex but controlled responses to input [8] that resemble activity patterns observed in the motor cortex. In particular, the dynamical state of the network plays a crucial role during the involved learning process. However, the effect of the input on the dynamical state has remained obscure.

To investigate the generic influence of external input on the network state, we include additive white noise in the seminal model by Sompolinsky et al. [1] and develop the dynamic mean-field theory for the resulting stochastic continuous-time dynamics. In contrast to the original work, here we reformulate the problem in terms of the generating functional formalism for stochastic differential equations [25-29]. The application of the auxiliary field formulation known from large $N$ field theory [30] then allows us

to derive the mean-field equations by a saddle-point approximation. As in the original model, the autocorrelation function is formally identical to the motion of a classical particle in a potential [1]. In our model, the noise amounts to an initial kinetic energy. We then determine the maximum Lyapunov exponent [31] by considering two copies of the system with different initial conditions [32] in a replica calculation. Our main result is a closed-form condition for the transition from stable to chaotic dynamics. We find that the input suppresses chaos significantly more strongly than expected from time-local linear stability, the criterion valid in discrete-time systems. This observation is explained by a dynamic effect: The decrease of the maximum Lyapunov exponent is related to the sharpening of the autocorrelation function by the stochastic drive. The regime in the phase diagram between local instability, as indicated by the spectral radius of the Jacobian, and transition to chaos, corresponding to a positive maximum Lyapunov exponent, constitutes an as yet unreported dynamical regime that combines locally expansive dynamics with asymptotic stability. Moreover, in contrast to the autonomous case, the decay time of the autocorrelation function does not diverge at the transition. Its peak is strongly reduced by the input and occurs slightly above the critical coupling strength.

To study information-processing capabilities, we evaluate the capacity to reconstruct a past input signal by a linear readout of the present state, the so-called memory curve [33]. Dynamic mean-field theory and a replica calculation lead to a closed-form expression for the memory curve. We find that the memory capacity peaks within the expansive, nonchaotic regime, indicating that locally expansive, while asymptotically stable dynamics is beneficial to store input sequences in the dynamics of the neural network.

\section{DYNAMIC MEAN-FIELD EQUATION}

We study the continuous-time dynamics of a random network of $N$ neurons, whose states $x_{i}(t) \in \mathbb{R}, i=1, \ldots, N$, evolve according to the system of stochastic differential equations,

$$
\frac{d x_{i}}{d t}=-x_{i}+\sum_{j=1}^{N} J_{i j} \phi\left(x_{j}\right)+\xi_{i}(t) .
$$

The $J_{i j}$ are independent and identically Gaussian-distributed random coupling weights with zero mean and variance $g^{2} / N$, where the intensive gain parameter $g$ controls the recurrent coupling strength or, equivalently, the weight heterogeneity of the network. We further exclude self-coupling, setting $J_{i i}=0$. The time-varying inputs $\xi_{i}(t)$ are independent Gaussian white-noise processes with correlation functions $\left\langle\xi_{i}(t) \xi_{j}(s)\right\rangle=2 \sigma^{2} \delta_{i j} \delta(t-s)$. We choose the sigmoidal transfer function $\phi(x)=\tanh (x)$ so that, without input, for $\sigma=0$, the model agrees with the autonomous one studied in Ref. [1]. 
The dynamical system (1) contains two sources of randomness: the quenched disorder due to the random coupling weights and the temporally fluctuating drive. A particular realization of the random couplings $J_{i j}$ defines a fixed network configuration, and its dynamical properties usually vary between different realizations. For large network size $N$, however, certain quantities are self-averaging, meaning that their values for a typical realization can be obtained by an average over network configurations [34]. An important example is the population-averaged autocorrelation function.

Here, we derive a dynamic mean-field theory that describes the statistical properties of the system under the joint distribution of disorder, noise, and possibly random initial conditions in the limit of large network size $N \rightarrow \infty$. The theory can be derived via a heuristic "local chaos" assumption [35] or by using a generating functional formulation $[17,36]$. Here, we follow the latter approach because it casts the problem into the established language of statistical field theory for which a wealth of approximation techniques is available [37]. A mathematically rigorous proof uses large deviation techniques [38]. The general idea is that for large network size $N$, the local recurrent input $\sum_{j=1}^{N} J_{i j} \phi\left(x_{j}\right)$ in Eq. (1) approaches a Gaussian process with self-consistently determined statistics.

We interpret the stochastic differential equations in the Itô convention [39] and formulate the problem (1) in terms of a moment-generating functional $Z$. Using the MartinSiggia-Rose-de Dominicis-Janssen path integral formalism $[25,28,40,41]$, we obtain

$$
Z[\mathbf{l}](\mathbf{J})=\int \mathcal{D} \mathbf{x} \int \mathcal{D} \tilde{\mathbf{x}} \exp \left(S_{0}[\mathbf{x}, \tilde{\mathbf{x}}]-\tilde{\mathbf{x}}^{\mathrm{T}} \mathbf{J} \phi(\mathbf{x})+\mathbf{l}^{\mathrm{T}} \mathbf{x}\right)
$$

with

$$
S_{0}[\mathbf{x}, \tilde{\mathbf{x}}]=\tilde{\mathbf{x}}^{\mathrm{T}}\left(\partial_{t}+1\right) \mathbf{x}+\sigma^{2} \tilde{\mathbf{x}}^{\mathrm{T}} \tilde{\mathbf{x}}
$$

where the field $\mathbf{x}=\left(x_{i}(t), t \in \mathbb{R}\right)_{i=1}^{N}$ is the vector of trajectories (paths), $\mathbf{x}^{\mathrm{T}} \mathbf{y}=\sum_{i} \int x_{i}(t) y_{i}(t) d t$ denotes the scalar product in time and in neuron space, and $\mathbf{l}=\left(l_{i}(t), t \in\right.$ $\mathbb{R})_{i=1}^{N}$ represents a source field. The response field $\tilde{\mathbf{x}}=\left(\tilde{x}_{i}(t), t \in \mathbb{R}\right)_{i=1}^{N}$ appears as a result of a HubbardStratonovich transformation, representing, at each time point, a Dirac delta as $\delta(x)=(2 \pi \mathrm{i})^{-1} \int_{a-\mathrm{i} \infty}^{a+\mathrm{i} \infty} d \tilde{x} \mathrm{e}^{\tilde{x} x}[29,41]$. The measures are defined as $\int \mathcal{D} \mathbf{x}=\lim _{M \rightarrow \infty} \Pi_{k=1}^{N} \Pi_{l=1}^{M} \int_{-\infty}^{\infty} d x_{k}^{l}$ and $\int \mathcal{D} \tilde{\mathbf{x}}=\lim _{M \rightarrow \infty} \Pi_{k=1}^{N} \Pi_{l=1}^{M} \int_{-\mathrm{i} \infty}^{\mathrm{i} \infty}(2 \pi \mathrm{i})^{-1} d \tilde{x}_{k}^{l}$, with the subscript $k$ denoting the $k$ th unit and the superscript $l$ denoting the $l$ th time slice. The action $S_{0}$ in Eq. (3) contains all singleunit properties, therefore excluding the coupling term $-\tilde{\mathbf{x}}^{\mathrm{T}} \mathbf{J} \phi(\mathbf{x})$, which is written explicitly in Eq. (2).
Assuming that the dynamics is self-averaging, we average over the quenched disorder in the coupling matrix $\mathbf{J}=\left(J_{i j}\right)_{i, j=1}^{N}$ and perform a saddle-point approximation (Appendix A). The resulting functional factorizes into $N$ terms

$\bar{Z}^{*} \propto \int \mathcal{D} x \int \mathcal{D} \tilde{x} \exp \left(S_{0}[x, \tilde{x}]+\frac{g^{2}}{2} \tilde{x}^{\mathrm{T}} C_{\phi(x) \phi(x)} \tilde{x}\right)$

with $C_{\phi(x) \phi(x)}(t, s)=\langle\phi(x(t)) \phi(x(s))\rangle$ denoting the average autocorrelation function of the nonlinearly transformed activity of the units, see Eq. (A10), and $\tilde{x}^{\mathrm{T}} C_{\phi(x) \phi(x)} \tilde{x}=\iint \tilde{x}(t) C_{\phi(x) \phi(x)}(t, s) \tilde{x}(s) d t d s$. The factorization reduces the network to $N$ noninteracting units, each on a background of an independent Gaussian noise with identical, self-consistently determined statistics. At this level of approximation, the problem is hence equivalent to a single-unit system. The effective equation of motion corresponding to this system can be read from Eq. (4) (Appendix A):

$$
\frac{d x}{d t}=-x+\eta(t)+\xi(t) .
$$

Without the additional input $\xi(t)$, Eq. (5) is the starting point of the analysis in Ref. [1]. Here, $\xi(t)$ is a Gaussian white-noise process as in Eq. (1), independent of $\eta(t)$. The centered Gaussian process $\eta(t)$ is fully specified by its autocorrelation function

$$
\langle\eta(t) \eta(s)\rangle=g^{2} C_{\phi(x) \phi(x)}(t, s) .
$$

\section{EFFECTIVE EQUATION OF MOTION OF THE AUTOCORRELATION FUNCTION}

Our goal is to determine the mean-field autocorrelation function $\langle x(t) x(s)\rangle$, which, by self-averaging, also describes the population-averaged autocorrelation function. Assuming that $x(t)$ is a stationary process, $c(\tau)=\langle x(t+$ $\tau) x(t)\rangle$ obeys the differential equation (Appendix B)

$$
\ddot{c}=\frac{d^{2} c}{d \tau^{2}}=c-g^{2} f_{\phi}\left(c, c_{0}\right)-2 \sigma^{2} \delta(\tau)
$$

with $c_{0}=c(0)$. The Dirac delta inhomogeneity originates from the white-noise autocorrelation function of the timevarying input. Without the delta inhomogeneity, Eq. (7) has been derived in Ref. [1], while the first derivation including the inhomogeneity, to our knowledge, has been presented by Cabana and Touboul [38], using the mathematically rigorous framework of large deviations. The same inhomogeneity arises from Poisson spiking noise with $2 \sigma^{2}=$ $g^{2} r$ [42], where $r$ is the population-averaged firing rate. One may obtain the same result by dynamic meanfield theory [43]. In Eq. (7), we write $f_{\phi}\left(c(\tau), c_{0}\right)=$ $C_{\phi(x) \phi(x)}(t+\tau, t)$, introducing the notation 
$f_{u}\left(c, c_{0}\right)=\iint u\left(\sqrt{c_{0}-\frac{c^{2}}{c_{0}}} z_{1}+\frac{c}{\sqrt{c_{0}}} z_{2}\right) u\left(\sqrt{c_{0}} z_{2}\right) D z_{1} D z_{2}$

for an arbitrary function $u(x)$, where $D z_{i}=\exp \left(-z_{i}^{2} / 2\right) /$ $\sqrt{2 \pi} d z_{i}$ denotes the standard Gaussian integration measure. This representation holds since $x(t)$ is itself a Gaussian process. Note that Eq. (8) reduces to a one-dimensional integral for $f_{u}\left(c_{0}, c_{0}\right)=\left\langle u\left(\sqrt{c_{0}} z_{1}\right)^{2}\right\rangle$ and $f_{u}\left(0, c_{0}\right)=$ $\left\langle u\left(\sqrt{c_{0}} z_{1}\right)\right\rangle^{2}$.

Following the approach in Ref. [1], we formulate Eq. (7) as the one-dimensional motion of a classical particle in a potential:

$$
\ddot{c}=-V^{\prime}(c)-2 \sigma^{2} \delta(\tau)
$$

where we define

$$
V(c)=V\left(c ; c_{0}\right)=-\frac{1}{2} c^{2}+g^{2} f_{\Phi}\left(c, c_{0}\right)-g^{2} f_{\Phi}\left(0, c_{0}\right)
$$

with $\Phi(x)=\int_{0}^{x} \phi(y) d y$ and $\partial / \partial c f_{\Phi}\left(c, c_{0}\right)=f_{\phi}\left(c, c_{0}\right)$ following from Price's theorem $[44,45]$. The autocorrelation $c(\tau)$ here plays the role of the position of the particle and the time lag $\tau$ the role of time. The potential (10) depends on the initial value $c_{0}$, which has to be determined self-consistently. We obtain $c_{0}$ from classical energy conservation, $\dot{c}^{2} / 2+V(c)=$ constant. Considering $\tau \geq 0$ and the symmetry of $c(\tau)$, the delta inhomogeneity in Eq. (9) amounts to an initial velocity $\dot{c}(0+)=-\sigma^{2}$ and thus to the kinetic energy $\dot{c}^{2}(0+) / 2=\sigma^{4} / 2$. Since $|c(\tau)| \leq c_{0}$, the solution $c(\tau)$ and its first derivative must approach zero as $\tau \rightarrow \infty$. Thus, we obtain the selfconsistency condition for $c_{0}$ as

$$
\frac{1}{2} \sigma^{4}+V\left(c_{0} ; c_{0}\right)=V\left(0 ; c_{0}\right)=0
$$

For the autonomous case, Figs. 1(c) and 1(e) show the resulting potential and the corresponding self-consistent autocorrelation function $c(\tau)$ in the chaotic regime. Approaching the transition from above, $g \rightarrow g_{c}=1$, the amplitude $c_{0}$ vanishes and the decay time of $c(\tau)$ given by $\tau_{\infty}=1 / \sqrt{1-g^{2}\left\langle\phi^{\prime}(x)\right\rangle^{2}}$ diverges [1]. This picture breaks down in the driven case [Figs. 1(d) and 1(f)], where $c_{0}$ is always nonzero and $c(\tau)$ decays with finite timescale and has a kink at zero. The mean-field prediction is in excellent agreement with the population-averaged autocorrelation function obtained from numerical simulations of one network instance showing that the self-averaging property is fulfilled. In the following, we derive a condition for the transition from stable to chaotic dynamics in the presence of the time-varying input.

\section{EFFECT OF INPUT ON THE TRANSITION TO CHAOS}

The maximum Lyapunov exponent quantifies how sensitively the dynamics depends on the initial conditions [31]. It measures the asymptotic growth rate of infinitesimal perturbations. For stochastic dynamics, the stability of the solution for a fixed realization of the noise or, equivalently, the stochastic input is also characterized by the maximum Lyapunov exponent [46]: If it is negative, trajectories with nearby initial conditions converge to the same timedependent solution determined by the input; the dynamics is stable. If it is positive, the distance between two initially arbitrary close trajectories grows exponentially in time; the dynamics exhibits sensitive dependence on initial conditions and is hence chaotic.

We derive the maximum Lyapunov exponent by using dynamic mean-field theory. To this end, we consider two copies of the network distinguished by superscripts $\alpha \in\{1,2\}$. These copies, or replicas, have an identical coupling matrix $\mathbf{J}$ and, for $\sigma>0$, are subject to the same realization of the stochastic inputs $\xi_{i}(t)$. The maximum Lyapunov exponent can be defined as the asymptotic growth rate of the Euclidean distance between trajectories of the two copies:

$$
\lambda_{\max }=\lim _{t \rightarrow \infty} \lim _{\left\|\mathbf{x}^{1}(0)-\mathbf{x}^{2}(0)\right\| \rightarrow 0} \frac{1}{2 t} \ln \left(\frac{\left\|\mathbf{x}^{1}(t)-\mathbf{x}^{2}(t)\right\|^{2}}{\left\|\mathbf{x}^{1}(0)-\mathbf{x}^{2}(0)\right\|^{2}}\right) .
$$

We now follow an idea by Derrida and Pomeau [32] and exploit the self-averaging property of population-averaged correlation functions, i.e., $(1 / N) \sum_{i=1}^{N} x_{i}^{\alpha}(t) x_{i}^{\beta}(s) \approx c^{\alpha \beta}(t, s)$, where $c^{\alpha \beta}(t, s)$ denotes the mean-field correlation functions. We express the mean-squared Euclidean distance as

$\frac{1}{N} \sum_{i=1}^{N}\left(x_{i}^{1}(t)-x_{i}^{2}(t)\right)^{2} \approx c^{11}(t, t)+c^{22}(t, t)-2 c^{12}(t, t)=d(t)$,

where we define the mean-field squared distance $d(t)$. Thus, the asymptotic growth rate of $d(t)$ provides us with a mean-field description of the maximum Lyapunov exponent. To obtain this growth rate, we first consider

$d(t, s)=c^{11}(t, s)+c^{22}(t, s)-c^{12}(t, s)-c^{21}(t, s)$

with the obvious property $d(t)=d(t, t)$. We then determine the temporal evolution of $d(t, s)$ for infinitesimally perturbed initial conditions $\left\|\mathbf{x}^{1}(0)-\mathbf{x}^{2}(0)\right\|=\epsilon$. To this end, it is again convenient to use a generating functional that captures the joint statistics of the two systems and, in addition, allows averaging over the quenched disorder (see also Ref. [37], last remark in Appendix 23). The generating 
functional describing the two copies is defined analogously to the single system functional (2) as

$$
\begin{aligned}
Z\left[\mathbf{l}^{1}, \mathbf{l}^{2}\right](\mathbf{J})= & \Pi_{\alpha=1}^{2}\left\{\int \mathcal { D } \mathbf { x } ^ { \alpha } \int \mathcal { D } \tilde { \mathbf { x } } ^ { \alpha } \operatorname { e x p } \left(S_{0}\left[\mathbf{x}^{\alpha}, \tilde{\mathbf{x}}^{\alpha}\right]\right.\right. \\
& \left.\left.-\tilde{\mathbf{x}}^{\alpha \mathrm{T}} \mathbf{J} \phi\left(\mathbf{x}^{\alpha}\right)+\mathbf{l}^{\alpha \mathrm{T}} \mathbf{x}^{\alpha}\right)\right\} \exp \left(2 \sigma^{2} \tilde{\mathbf{x}}^{1 \mathrm{~T}} \tilde{\mathbf{x}}^{2}\right),
\end{aligned}
$$

with the single-system "free action" $S_{0}[\mathbf{x}, \tilde{\mathbf{x}}]$ defined in Eq. (3). The factor in the last line results from the identical external input in the two copies and effectively couples the two systems. We also note that the coupling matrix $\mathbf{J}$ is the same in both copies.

Averaging Eq. (13) over the quenched disorder of the random coupling matrix $\mathbf{J}$ and performing a saddle-point approximation, we obtain a pair of effective equations of motion (Appendix C),

$$
\left(\frac{d}{d t}+1\right) x^{\alpha}(t)=\xi(t)+\eta^{\alpha}(t), \quad \alpha \in\{1,2\},
$$

together with a set of self-consistency equations for the correlations of the Gaussian noises $\eta^{\alpha}(t)$,

$$
\left\langle\eta^{\alpha}(t) \eta^{\beta}(s)\right\rangle=g^{2}\left\langle\phi\left(x^{\alpha}(t)\right) \phi\left(x^{\beta}(s)\right)\right\rangle .
$$

Now, there are two terms that introduce correlations between the two copies. First, the common fluctuating drive $\xi(t)$ is injected into both systems. Second, the effective noises $\eta^{\alpha}(t)$ and $\eta^{\beta}(t)$ can be correlated between replicas, see Eq. (15), because the two systems have the same coupling $\mathbf{J}$ in each realization. The origin of the latter coupling is hence of static nature.

The squared distance (12) between the two copies is given by the autocorrelations of the single systems and the cross-correlations between them. We consider the case where both copies are prepared with identical initial conditions and thus are fully synchronized: The crosscorrelation $c^{12}(t, s)$ initially equals the autocorrelations $c^{11}(t, s), c^{22}(t, s)$. The latter are identical to the singlesystem autocorrelation function because the marginal statistics of each subsystem is not affected by the mere presence of the other system. An increase of the squared distance $d(t)$, by Eq. (12), amounts to a decline of $c^{12}(t, s)$ away from its initial value $c(t-s)$. Here, $c$ is the stationary autocorrelation, as we are interested in the Lyapunov exponent averaged over initial conditions drawn from the stationary distribution. To determine the growth rate for infinitesimal distances $d(0) \propto \epsilon$ between the two copies, we therefore expand the cross-correlation around its stationary solution $c^{12}(t, s)=c(t-s)+\epsilon k^{(1)}(t, s)$, $\epsilon \ll 1$, which, to linear order, leads to an equation of motion for the deflection (Appendix C 1)

$$
\left(\partial_{t}+1\right)\left(\partial_{s}+1\right) k^{(1)}(t, s)=g^{2} f_{\phi^{\prime}}\left(c(t-s), c_{0}\right) k^{(1)}(t, s)
$$

with $d(t)=-2 \epsilon k^{(1)}(t, t)$.

A separation ansatz in the coordinates $\tau=t-s$ and $T=$ $t+s$ then yields an eigenvalue problem in the form of a timeindependent Schrödinger equation [1,42] (Appendix C 2),

$$
\left[-\partial_{\tau}^{2}+W(\tau)\right] \psi(\tau)=E \psi(\tau)
$$

where $\tau$ now plays the role of a spatial coordinate. Sompolinsky et al. [1] state this Schrödinger equation for the Lyapunov exponent, but no details are provided. As in their case, the quantum potential $W(\tau)=-V^{\prime \prime}(c(\tau))=1-$ $g^{2} f_{\phi^{\prime}}\left(c(\tau), c_{0}\right)$ is the negative second derivative of the classical potential $V(c)$ evaluated along the self-consistent autocorrelation function $c(\tau)$. The ground-state energy $E_{0}$ of Eq. (17) determines the asymptotic growth rate of $k^{(1)}(t, t)$ as $t \rightarrow \infty$ and, hence, the maximum Lyapunov exponent via $\lambda_{\max }=-1+\sqrt{1-E_{0}}$. Therefore, the dynamics is predicted to become chaotic if $E_{0}<0$. The quantum potential, together with the solution for the ground-state energy and wave function, is shown in Fig. 2. The latter are obtained as solutions of a finite-difference discretization of Eq. (17).

In the autonomous case, a decaying autocorrelation function corresponds to a positive maximum Lyapunov exponent [1]. This follows from the observation that for $g>1$ the derivative of the self-consistent autocorrelation function $\dot{c}(\tau)$ solves the Schrödinger equation with $E=0$. But as $\dot{c}(\tau)$ is an eigenfunction with a single node, it cannot be the ground state, which must have zero nodes. The ground-state energy, which is necessarily lower, must therefore be negative, $E_{0}<0$. So the dynamics is chaotic, and $\lambda_{\max }$ crosses zero at $g=1$ [Fig. 3(a)].
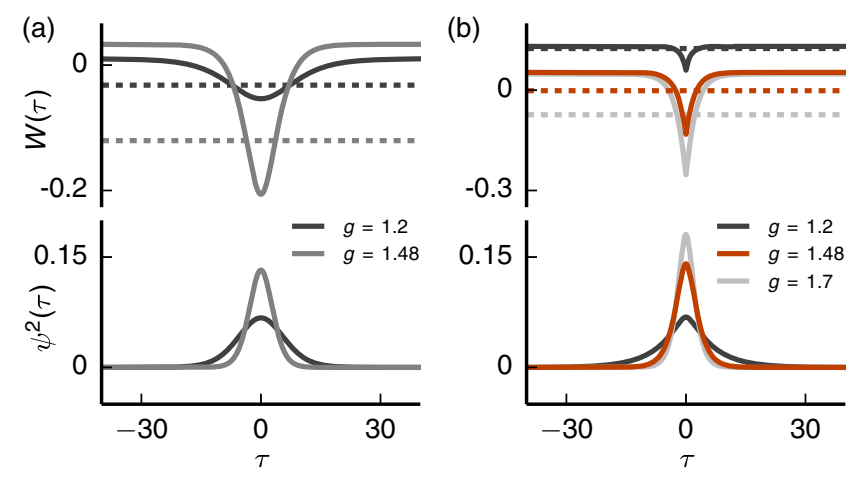

FIG. 2. Ground state of the Schrödinger equation, which determines the Lyapunov exponent. Upper part of vertical axis: Quantum potential $W(\tau)$ (solid curve) and ground-state energy $E_{0}$ (dashed line) for the autonomous case (a) and the driven case (b) for $\sigma=0.35$. Lower part of vertical axis: Corresponding squared ground-state wave function. The parameters are the same as in Fig. 1 (with the driven case for $g=0$ left out). 

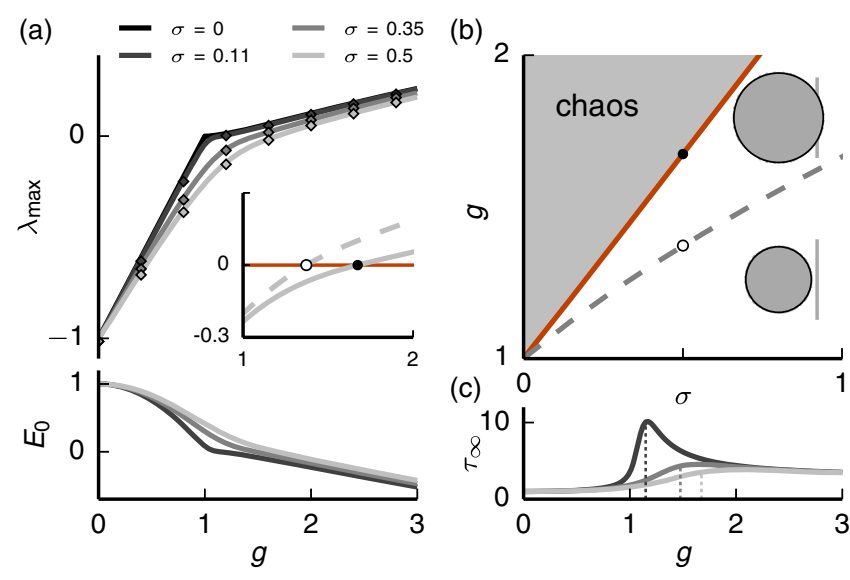

FIG. 3. Transition to chaos. (a) Upper part of vertical axis: Maximum Lyapunov exponent $\lambda_{\max }$ as a function of the coupling strength $g$ for different input amplitude levels. Mean-field prediction (solid curve) and simulation results (diamonds). We show a comparison to the upper bound $-1+g \sqrt{\left\langle\left[\phi^{\prime}(x)\right]^{2}\right\rangle}$ (dashed curve) for $\sigma=0.5$ in the inset. Zero crossings are marked with dots. Lower part of vertical axis: Ground-state energy $E_{0}$ as a function of $g$. (b) Phase diagram with transition curve (solid red curve) obtained from Eq. (20) and necessary condition [Eq. (18) with equal sign] (gray dashed curve). Dots correspond to zero crossings in the inset in panel (a). We show the disk of eigenvalues of the Jacobian matrix in Eq. (19) for $\sigma=0.8$ and $g=1.25$ (lower) and $g=2.0$ (upper) centered at -1 in the complex plane (gray dots). The radius $\rho=g \sqrt{\left\langle\left[\phi^{\prime}(x)\right]^{2}\right\rangle}$ is obtained from random matrix theory (black circle). The vertical line is at zero. (c) Asymptotic decay time $\tau_{\infty}$ of the autocorrelation function. Vertical dashed lines mark the transition to chaos. The color code is the same as in panel (a). The network size in the simulations is $N=5000$.

In the presence of a fluctuating drive, the maximum Lyapunov exponent becomes positive at a critical coupling strength $g_{c}>1$; with increasing input amplitude, the transition shifts to larger values [Fig. 3(a)]. The mean-field prediction $\lambda_{\max }=-1+\sqrt{1-E_{0}}$ shows excellent agreement with the Lyapunov exponent obtained in simulations using a standard algorithm [31]. Since the ground-state energy $E_{0}$ must be larger than the minimum $W(0)=1-$ $g^{2}\left\langle\left[\phi^{\prime}(x)\right]^{2}\right\rangle$ of the quantum potential, an upper bound for $\lambda_{\max }$ is provided by $-1+g \sqrt{\left\langle\left[\phi^{\prime}(x)\right]^{2}\right\rangle}$, leading to a necessary condition

$$
\rho=g \sqrt{\left\langle\left[\phi^{\prime}(x)\right]^{2}\right\rangle} \geq 1
$$

for chaotic dynamics. However, close to the transition, $\lambda_{\max }$ is clearly smaller than the upper bound, which is a good approximation only for small $g$ [Fig. 3(a), inset]: The actual transition occurs at substantially larger coupling strengths. In contrast, for memoryless discrete-time dynamics, the necessary condition found here is also sufficient for the transition to chaos [see Eq. (13) in Ref. [23] ].
The local linear stability of the dynamical system (1) is analyzed via the variational equation

$$
\frac{d}{d t} y_{i}(t)=-y_{i}(t)+\sum_{j=1}^{N} J_{i j} \phi^{\prime}\left(x_{j}(t)\right) y_{j}(t),
$$

$i=1, \ldots, N$, describing the temporal evolution of an infinitesimal deviation $y_{i}(t)$ about a reference trajectory $x_{i}(t)$. Interestingly, $\rho=g \sqrt{\left\langle\left[\phi^{\prime}(x)\right]^{2}\right\rangle}$ in Eq. (18) is also the radius of the disk formed by the eigenvalues of the Jacobian matrix in the variational equation (19) estimated by random matrix theory $[21,22]$. In the following, we refer to $\rho$ as the eigenvalue radius. Therefore, the dynamics is expected to become locally unstable if this radius exceeds unity, as shown in Fig. 3(b) displaying $\rho$ and the eigenvalues at an arbitrary point in time. But even for the case with $\rho>1$, the system is not necessarily chaotic. Hence, contrary to the autonomous case [1,21], the transition to chaos is not predicted by random matrix theory.

To derive an exact condition for the transition, we determine a ground state with vanishing energy $E_{0}=0$. As in the autonomous case, $\dot{c}(\tau)$ solves Eq. (17) for $E=0$, except at $\tau=0$ where it exhibits a jump, because $c(\tau)$ has a kink due to the white-noise input, Eq. (7). By linearity, $|\dot{c}(\tau)|$ is a continuous and symmetric solution with zero nodes. Therefore, if its derivative is continuous as well, requiring $\ddot{c}(0+)=0$, it constitutes the ground state that we searched for. This is in contrast to the autonomous case, where $\dot{c}(\tau)$ corresponds to the first excited state. Consequently, with Eq. (7), we find the condition for the transition

$$
g_{c}^{2} f_{\phi}\left(c_{0}, c_{0}\right)-c_{0}=0,
$$

in which $c_{0}$ is determined by the self-consistency condition (11) resulting in the transition curve $\left(g_{c}, \sigma_{c}\right)$ in parameter space [Fig. 3(b)]. This reveals the relationship between the onset of chaos, the statistics of the random coupling matrix, and the input amplitude.

From Eq. (20), it follows that the system becomes chaotic precisely when the variance $c_{0}$ of a typical single unit equals the variance of its recurrent input from the network $g^{2}\left\langle\phi^{2}(x)\right\rangle$. At the transition, the classical selfconsistent potential $V\left(c ; c_{0}\right)$ has a horizontal tangent at $c_{0}$, while in the chaotic regime, a minimum emerges [Fig. 1(d)]. With Eq. (7) it follows that the curvature $\ddot{c}(0+)$ of the autocorrelation function at zero changes sign from positive to negative [Fig. 1(f)]. Close to the transition, a standard perturbative approach shows that $\lambda_{\max }$ is proportional to $g^{2}\left\langle\phi^{2}(x)\right\rangle-c_{0}$, indicating a self-stabilizing effect: Since both terms grow with $g$, the growth of their difference is attenuated, explaining why $\lambda_{\max }(g)$ bends down as the transition is approached [Fig. 3(a)]. 
In the autonomous case, the timescale of fluctuations diverges at the transition to chaos [1]. Here, we consider the effect of the input on the asymptotic decay time $\tau_{\infty}$ of the autocorrelation function [Fig. 3(c)]. For weak input amplitude, the decay time peaks at the transition, corresponding to the diverging timescale in the autonomous case. For larger input amplitudes, the peak is strongly reduced, and the maximum decay time is attained above the transition.

\section{STATIC AND DYNAMIC SUPPRESSION OF CHAOS}

The condition (20) predicts the transition at significantly larger coupling strengths than the necessary condition $\rho=$ $g \sqrt{\left\langle\left[\phi^{\prime}(x)\right]^{2}\right\rangle} \geq 1$ [Fig 3(b)]. In this section, we show that chaos suppression can be decomposed into a static and a dynamic mechanism. We first consider the static effect, which can be fully attributed to the increase of the variance $c_{0}$ caused by the additional input. Because $\phi^{\prime}(x)$ is maximal at the origin, increasing the variance $c_{0}$ reduces the averaged squared slope in $g^{2}\left\langle\left[\phi^{\prime}(x)\right]^{2}\right\rangle$ with $x \sim$ $\mathcal{N}\left(0, c_{0}\right)$, thereby stabilizing the dynamics. The same effect can be obtained if we replace the white-noise input in Eq. (1) by a static (constant in time) heterogeneous input $\xi_{i} \stackrel{\text { i.i.d. }}{\sim} \mathcal{N}\left(0, \sigma_{\mathrm{q}}^{2}\right)$, called quenched noise in the following. In this case, the calculation of the saddle-point solution, analogous to Sec. III, leads to the differential equation (Appendix D)

$$
\ddot{c}=c-g^{2} f_{\phi}\left(c, c_{0}\right)-\sigma_{\mathrm{q}}^{2}
$$

for the stationary autocorrelation function $c(\tau)$.

A possible self-consistent solution of Eq. (21) is given by a constant autocorrelation function $c(\tau)=c_{0}$, which describes the variance $c_{0}$ of a heterogeneous fixed point of the network dynamics in the presence of heterogeneous static input. Such a fixed point loses stability when the eigenvalue radius of the Jacobian matrix $\rho=g \sqrt{\left\langle\left[\phi^{\prime}(x)\right]^{2}\right\rangle}$ evaluated at the fixed point exceeds unity. This determines a critical coupling strength, which coincides with our necessary condition. Hence, we denote its value by $g_{\text {nec }}$.

For $g>g_{\text {nec }}$, a decaying autocorrelation function $c(\tau)$ emerges which decays to a nonzero asymptotic value $c_{\infty}$ (Appendix D). The function can be constructed by again writing Eq. (21) in the form of a classical equation of motion in a potential: $\ddot{c}=-V_{\mathrm{q}}{ }^{\prime}(c)$. The modified potential $V_{\mathrm{q}}\left(c ; c_{0}\right)$ is given by Eq. (10) plus the additional linear term $\sigma_{\mathrm{q}}^{2} c$ originating from the quenched noise. The asymptotic value requires $V_{\mathrm{q}}{ }^{\prime}\left(c_{\infty} ; c_{0}\right)=0$, which we solve together with classical energy conservation, $V_{\mathrm{q}}\left(c_{\infty} ; c_{0}\right)=$ $V_{\mathrm{q}}\left(c_{0} ; c_{0}\right)$, to self-consistently determine the initial value $c_{0}$ [Fig. 4(a)]. By integrating Eq. (21), we obtain the corresponding self-consistent autocorrelation function $c(\tau)$, which agrees with direct simulations [Fig. 4(b)].
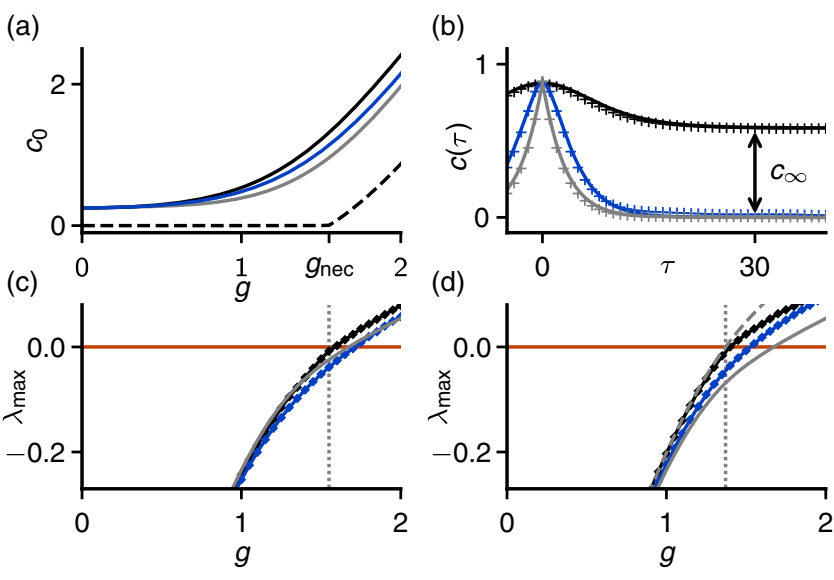

FIG. 4. Effect of correlation time of the input on the transition to chaos. The cases of white-noise, colored noise with $\tau_{\mathrm{n}}=2$, and quenched input correspond to the curves shown in gray, blue, and black, respectively. (a) Self-consistent variance $c_{0}$ depending on the coupling $g$. At vanishing coupling, the inputs are chosen such that $c_{0}=\sigma^{2}=0.25$ is identical in all three cases of white, colored, and quenched noise. Resulting dynamic contribution $c_{0}-c_{\infty}$ (dashed black curve) of the variance in the case of quenched noise. (b) Self-consistent autocorrelation function (solid curves) compared to simulations (crosses) for $g=1.5$. The amplitude of colored and quenched noise is adjusted to obtain the same variance (peak height) as for white noise. (c) Maximum Lyapunov exponent as a function of $g$ for colored and quenched noise measured in simulations (diamonds connected by lines) compared to white noise (identical to Fig. 3(a) with $\sigma=0.5$ ). (d) Same as in panel (c) but for identical variance $c_{0}$ for each value of $g$. Gray curves correspond to the Lyapunov exponent (solid) and the upper bound (dashed) from the white-noise case. The necessary condition (18) is marked as a gray dotted vertical line in panels (c) and (d).

The Schrödinger equation for the Lyapunov exponent (17) also holds in the case of quenched-noise input since the explicit form of the noise term vanishes in the linearized equation (16) (Appendix D). As in the case without input [1], $\dot{c}(\tau)$ is an eigenfunction with a single node, and therefore, the dynamics corresponding to a decaying autocorrelation function must be chaotic; we hence conclude that the transition to chaos for quenched input occurs at $g_{\text {nec }}$, which is confirmed by the numerically estimated maximum Lyapunov exponent [Fig 4(c)].

In the following, we consider how the variance $c_{0}$ depends on $g$ for the different cases of white- and quenched-noise input. This is important because many of the relevant quantities like the eigenvalue radius $\rho$ depend on $c_{0}$. For $g=0$, we have $c_{0}=\sigma_{\mathrm{q}}^{2}$; so, choosing $\sigma_{\mathrm{q}}^{2}=\sigma^{2}$ leads to the same variance $c_{0}$ as for the white-noise input in Eq. (1). With increasing coupling strength $g$, however, the variance $c_{0}$ grows significantly faster for quenched-noise input [Fig. 4(a)]: The resulting internally generated fluctuations are stronger in this case, which will have important consequences for information-processing capabilities as we investigate below in Sec. VII. 
To isolate the dynamic effect on the transition to chaos, we need to keep the static effect identical for quenched- and white-noise input. To maintain the same variance $c_{0}$ also for nonzero couplings, we decrease the input variance $\sigma_{\mathrm{q}}^{2}$ with increasing $g$. Because the variance $c_{0}$ is now identical in both cases, the eigenvalue radius is identical, too, as is the minimum of the quantum mechanical potential in Eq. (17).

Comparing the Lyapunov exponents in the two cases shows that the system with quenched input becomes chaotic at a smaller coupling than the system with white-noise input [Fig. 4(d)]. As expected, the point of the transition coincides with the critical value $g_{\text {nec }}$. Therefore, a dynamical regime between the loss of local stability and the transition to chaos does not exist in the case of quenched input.

The comparison between quenched- and white-noise input demonstrates two points: First, the variability of the input, be it static or dynamic, increases the variance $c_{0}$. It therefore reduces the eigenvalue radius $\rho$ and increases the minimum of the quantum mechanical potential and thus suppresses chaos by a purely static effect that is identical in both cases. This mechanism corresponds to the one observed for discrete-time dynamics [9,23]. Second, the remaining difference between the Lyapunov exponents in Fig. 4(d) for quenched input and white-noise input is a dynamic effect caused by the temporal variations of the input. It occurs for the continuous-time dynamics considered here and is also expected to appear in discrete-time networks with leaky single-unit dynamics.

To gain some insight into this dynamic mechanism, we return to the variational equation (19) describing the evolution of infinitesimal perturbations. Its fundamental solution can be written as a product of short-time propagator matrices:

$$
\begin{aligned}
\mathbf{y}(t) & =\exp \left(\int_{0}^{t}\left[-1+\mathbf{J} \phi^{\prime}(\mathbf{x}(s))\right] d s\right) \mathbf{y}(0) \\
& =\lim _{h \rightarrow 0} \prod_{l=0}^{t / h-1}\left[1-h+h \mathbf{J} \phi^{\prime}(\mathbf{x}(l h))\right] \mathbf{y}(0),
\end{aligned}
$$

where the matrix $\mathbf{J} \phi^{\prime}(\mathbf{x})$ has the entries $\left(\mathbf{J} \phi^{\prime}(\mathbf{x})\right)_{i j}=$ $J_{i j} \phi^{\prime}\left(x_{j}\right)$. Here, each factor has the same stability properties since the fraction of its eigenvalues with positive real parts stays approximately constant. However, the corresponding unstable directions may vary in time: They are determined by the matrix $\mathbf{J} \phi^{\prime}(\mathbf{x}(l h))$ at the respective discretized time point $l h$. The rate of this variation depends on the timescale on which $\phi^{\prime}(x(t))$ varies, which for fixed $c_{0}$ is monotonically related to the decay of the autocorrelation function $c(\tau)$. We conjecture that a faster variation of the unstable directions as indicated by a sharper autocorrelation function decreases the growth of generic perturbations. If this temporal variation is induced by time-varying input, it is even possible that perturbations shrink despite the presence of unstable directions at each point in time.

Is the dynamic mechanism also present for nonwhite time-varying input? How does the timescale of the autocorrelation function affect the transition? To answer these questions, we investigate the case of colored Gaussian noise inputs in the form of Ornstein-Uhlenbeck processes with finite correlation time (Appendix D). This setting constitutes an intermediate case between the two extremes discussed above. Again, we keep the variance $c_{0}$ at the same level as for white-noise input; hence, the static effect is identical in all three cases. The Lyapunov exponent for colored noise input lies between the two limiting cases [Fig. 4(d)], and the dynamic effect is still present as the transition to chaos occurs at a larger coupling strength compared to the case of quenched input. Indeed, Fig. 4(d) shows that for fixed $c_{0}$, the Lyapunov exponent decreases if the input varies faster, which is in line with a sharper autocorrelation function [Fig. 4(b)]. Note that for colored noise input, the autocorrelation function does not exhibit a kink at the origin, so this is not a prerequisite for the dynamic effect.

We can also understand the dynamic effect on the basis of the Schrödinger equation (17). As noted above, the form of the input only implicitly appears by shaping the quantum potential $W(\tau)=-V^{\prime \prime}(c(\tau))=1-g^{2} f_{\phi^{\prime}}\left(c(\tau), c_{0}\right)$, where $c(\tau)$ is the self-consistent autocorrelation function in the presence of the quenched or colored noise input. Increasing the correlation time of the input while keeping the variance $c_{0}$ constant leads to a slower decay of the autocorrelation function [Fig. 4(b)] and thereby to a wider quantum potential with identical minimum $W(0)$ because the latter only depends on $c_{0}$. Therefore, the ground-state energy decreases, and the Lyapunov exponent increases. The sign of the minimum also determines the local stability. So, for both cases of time-varying noise, white noise and colored noise, there is an offset between local stability and the transition to chaos [Fig. 4(d)]; an intermediate regime exists, and its extent decreases with increasing correlation time of the input. The term shaping the quantum potential is $f_{\phi^{\prime}}\left(c(\tau), c_{0}\right)=\left\langle\phi^{\prime}(x(t+\tau)) \phi^{\prime}(x(t))\right\rangle$, the autocorrelation function of $\phi^{\prime}(x(t))$. This directly links the temporal variation of $\phi^{\prime}(x(t))$ to the Lyapunov exponent and hence to the growth of generic perturbations as conjectured above.

In low-dimensional systems, the suppression of chaos by external fluctuations is understood: Noise forces the system to visit regions of the phase space with locally contracting dynamics more frequently [24], so contraction dominates expansion, in total yielding stable asymptotic behavior. This mechanism is similar to the static stabilization effect described above, where fluctuating drive causes the system to sample regions of the phase space with smaller eigenvalues of the Jacobian. The self-averaging high-dimensional system, however, has a constant eigenvalue radius over time, and hence, the dynamics is either 
locally contracting or locally expanding for all times. While the previous effects are explained by local stability, the dynamic suppression of chaos found here is a genuinely time-dependent mechanism, explained by the time evolution of the Jacobian in Eq. (22).

\section{NONVANISHING MEAN COUPLING AND NON-NEGATIVE TRANSFER FUNCTIONS}

The random coupling weights $J_{i j}$ with vanishing mean and the odd transfer function $\phi(x)=\tanh (x)$ in Eq. (1) are certainly not biologically realistic. We considered this case before to explain the mechanism of chaos suppression by fluctuating drive in the simplest setting; it allowed us to directly compare our findings to the classical results by Sompolinsky et al. [1]. In this section, we extend our analysis to the biologically more realistic case of random networks with nonvanishing mean coupling and with nonnegative transfer functions.

\section{A. Rectified-linear transfer function}

We first examine the rectified-linear transfer function $\phi(x)=\max (x, 0)$, for which the transition to chaos has been investigated recently $[20,42,43]$. However, the effect of time-varying input in these networks has not been studied yet. We consider Gaussian-distributed coupling weights $J_{i j} \stackrel{\text { i.i.d. }}{\sim} \mathcal{N}\left(\bar{g} / N, g^{2} / N\right)$ or the sparse connectivity of a directed Erdős-Rényi random network with connection probability $p$ and nonzero weight $J_{0} / \sqrt{N}$. Both cases lead to the same mean-field equations by identifying the parameters for the mean as $\bar{g}=\sqrt{N} J_{0}$ and the variance as $g^{2}=J_{0}^{2} p(1-p)$ (shown in Appendix E; see also Ref. [42]). The resulting equation for the autocorrelation function $c(\tau)$ is identical to Eq. (7), but with $f_{\phi(\circ+\langle x\rangle)}\left(c_{0}, c(\tau)\right)$ : We need to displace the transfer function by the nonzero mean of $x$ as $\phi(\circ+\langle x\rangle)$. The additional equation for the mean reads

$$
\langle x\rangle=\bar{g}\langle\phi(x)\rangle=\bar{g} \int D z \phi\left(\langle x\rangle+\sqrt{c_{0}} z\right),
$$

where $D z$ is again the standard Gaussian measure.

In structure, these equations resemble those that arise in the Sherrington-Kirkpatrick spin-glass model [see Eqs. (2.17) and (2.18) in Ref. [47] ], which is not surprising given that the physical problems are related and a similar mean-field approximation of the auxiliary fields has been employed. Importantly, $C_{\phi(x) \phi(x)}(\tau)=f_{\phi(\circ+\langle x\rangle)}\left(c_{0}, c(\tau)\right)$ approaches a nonzero asymptotic value for $\tau \rightarrow \infty$ (Appendix E). It reflects the variability of the average input across neurons in the network that arises from the variability of the incoming connections and the nonzero mean activity of each neuron. Qualitatively, it is the same as the addition of a quenched noise with variance $\sigma_{q}^{2}=g^{2} C_{\phi(x) \phi(x)}(\infty)$, which we have studied above (Sec. V). Correspondingly, the autocorrelation function $c(\tau)$ also approaches a nonzero asymptotic value $c_{\infty}$; we determine it simultaneously with the variance $c_{0}$ and the mean $\langle x\rangle$ by self-consistently solving Eq. (23) together with the condition for the asymptotic value $V^{\prime}\left(c_{\infty} ; c_{0}\right)=0$ and energy conservation (11). These statistics are in excellent agreement with direct simulations [inset in Fig 5(a)].

We now consider the effect of the nonvanishing mean coupling on the transition to chaos. The variability in the average input across neurons, as reflected by $c_{\infty}>0$, leads to an additional static contribution to the suppression of chaos as in the quenched-noise case treated in Sec. V. Does the dynamic suppression of chaos, as well as the intermediate regime between local instability and the transition to chaos, persist in this setting? The replica calculation proceeds completely analogously to the case of Gaussiandistributed couplings with vanishing mean (Appendix F). The result is a pair of effective equations of the form

$$
\left(\frac{d}{d t}+1\right) x^{\alpha}(t)=\bar{g}\left\langle\phi\left(x^{\alpha}(t)\right)\right\rangle+\xi(t)+\eta^{\alpha}(t),
$$

$\alpha \in\{1,2\}$, together with a set of self-consistency equations for the correlations of the noises $\eta^{\alpha}(t)$,

$$
\left\langle\eta^{\alpha}(t) \eta^{\beta}(s)\right\rangle=g^{2}\left\langle\phi\left(x^{\alpha}(t)\right) \phi\left(x^{\beta}(s)\right)\right\rangle .
$$

Compared to Eq. (14), the only difference is the term $\bar{g}\left\langle\phi\left(x^{\alpha}\right)\right\rangle$ in Eq. (24), which represents the nonzero mean input from the network. Importantly, the additional term
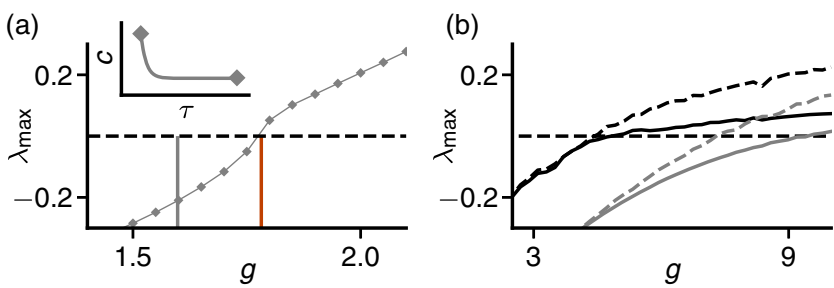

FIG. 5. Transition to chaos for nonvanishing mean coupling and more realistic transfer functions. (a) Rectified-linear transfer function $\phi(x)=\max (x, 0)$ : Maximum Lyapunov exponent as a function of $g$ measured in simulations (diamonds connected by lines), transition criterion (20) (red vertical line), and necessary condition (18) (gray vertical line) for $\bar{g}=-1$ and $\sigma=0.5$. The inset shows the autocorrelation function in simulations (solid curve) and $c_{0}, c_{\infty}$ (diamonds) from the self-consistent solution for $g=1.5$. (b) Leaky integrate-and-fire neuron transfer function: Maximum Lyapunov exponent (solid curve) and eigenvalue radius $\rho$ minus one (dashed curve) as a function of $g$ for the autonomous (black) and driven cases (gray). The network size is $N=1000$, and $\bar{g} \in[-0.05,-30]$ varies linearly with $g$. Other parameters are $J_{\text {ext }}=0.1, \nu_{\text {ext }}=600 \mathrm{~Hz}$, and $\sigma=7.1$. Parameters of the transfer function (for definitions, see, e.g., Eq. (27) in Ref. [48]) are as follows: $\tau_{\mathrm{m}}=20 \mathrm{~ms}$, synaptic time constant $\tau_{\mathrm{s}}=1 \mathrm{~ms}$, threshold $\theta=1$, reset $V_{\mathrm{r}}=0$, and refractory period $\tau_{\mathrm{r}}=2 \mathrm{~ms}$. Simulations were carried out with NEST [49]. 
does not introduce a coupling between the two replicas and therefore does not directly affect their correlation. It is determined independently for each replicon by a separate self-consistency equation of the form (23). Therefore, the mean coupling enters the calculation of the maximum Lyapunov exponent only indirectly by shaping the autocorrelation function: the shift of the transfer function $\phi(\circ+\langle x\rangle)$ in Eq. (7) and the nonzero asymptotic value $c_{\infty}$. Furthermore, the same transition criterion (20) still applies; this prediction is confirmed by direct simulations in Fig. 5(a). As a consequence, the dynamic mechanism of chaos suppression is identical to the case of coupling with vanishing mean, and the locally unstable but globally stable regime emerges [Fig. 5(a)].

\section{B. Leaky integrate-and-fire neuron transfer function}

Here, we demonstrate that the mechanism of dynamic chaos suppression is also present in random networks of biologically more realistic neuron models. To this end, we consider a nonlinear rate model $[43,50]$ :

$$
\frac{d \nu_{i}}{d t}=-\nu_{i}+\phi\left(\mu_{i}, \sigma_{i}\right)
$$

with

$$
\begin{aligned}
\mu_{i} & =\tau_{\mathrm{m}} \sum_{j} J_{i j} \nu_{j}+\tau_{\mathrm{m}} J_{\mathrm{ext}} \nu_{\mathrm{ext}}+\xi_{i}(t), \\
\sigma_{i}^{2} & =\tau_{\mathrm{m}} \sum_{j} J_{i j}^{2} \nu_{j}+\tau_{\mathrm{m}} J_{\mathrm{ext}}^{2} \nu_{\mathrm{ext}} .
\end{aligned}
$$

The transfer function $\phi$ in Eq. (26) is the firing rate function of the leaky integrate-and-fire (LIF) neuron model with synaptic filtering [51]. Each $\nu_{i}$ can be interpreted as the instantaneous firing rate of a LIF neuron (or an uncoupled population thereof), and Eq. (26) represents the rate dynamics of a random network of such neurons. More precisely, the firing rate response of these neurons is approximated by a first-order low-pass characteristic, qualitatively capturing the dominant behavior $[52,53]$.

As above, the coupling weights are Gaussian distributed, $J_{i j}$ i.i.d. $\mathcal{N}\left(\bar{g} / N, g^{2} / N\right)$. We also include a timehomogeneous external input to the neurons with firing rate $\nu_{\text {ext }}$ and weight $J_{\text {ext }}$, since this is usually present in biological networks. Here, $\tau_{\mathrm{m}}$ is the membrane time constant of the corresponding LIF neurons, and it sets the scale of the firing rates.

Our goal is to study the effect of the external timevarying drive $\xi_{i}(t)$ on the transition to chaos; it models an additional fluctuating input driving the membrane potential of the corresponding LIF neurons and therefore contributes to $\mu_{i}$ in Eq. (26). The Gaussian white-noise input in Eq. (1) is low-pass filtered before it enters the nonlinear transfer function. Consequently, for $\xi_{i}(t)$, we use independent
Gaussian colored noise with correlation time $\tau_{\mathrm{n}}=1$ and standard deviation $\sigma$.

Using simulations, we show that both the transition to chaos and the dynamic suppression of chaos exist in these networks [Fig. 5(b)]. To assess the local linear stability, we simulate Eq. (26) and determine the eigenvalue radius $\rho$ by numerically evaluating the eigenvalues of the Jacobian matrix $\left(\partial \phi_{i} / \partial \nu_{j}\right)$ [Eqs. (A.2) and (A.3) in Ref. [54] ]. The eigenvalue radius $\rho$ minus one and the maximum Lyapunov exponent $\lambda_{\max }$ both become positive when the variability of the couplings $g$ is increased [Fig. 5(b)]. To keep the transfer function from complete saturation, and thus the rates of the neurons in their dynamical range, we balance the increase in $g$ by a more negative mean coupling strength $\bar{g}$. For an autonomous network, i.e., for $\xi_{i} \equiv 0$, the transition to chaos precisely coincides with the local linear instability. Below the transition, the Lyapunov exponent $\lambda_{\max }$ equals $\rho-1$ as expected for a dynamical system at a stable fixed point. For the driven, nonautonomous case, however, a dynamical regime emerges where the eigenvalue radius $\rho$ exceeds unity, while the Lyapunov exponent $\lambda_{\max }$ is still negative. Hence, the mechanism of dynamic chaos suppression also persists in this more realistic setup.

\section{INFORMATION-PROCESSING CAPABILITIES}

We expect the expansive, nonchaotic regime to be beneficial for information processing: The local instability of the network ensures sufficient initial amplification of an impinging external signal. The asymptotic stability is required for the driving signal to be uncorrupted by the amplification of small variations of the input; it is hence necessary to ensure generalization. In the following, we investigate these ideas quantitatively by considering the sequential memory capacity of the network.

We focus on the component $z(t)=1 / N \sum_{i=1}^{N} \xi_{i}(t)$ of the input that is received by all neurons with equal strength. In other words, the total input to each neuron is decomposed into the signal $z(t)$ and the remaining inputs $\xi_{i}(t)-z(t)$, which act as noise. In Appendix G, we treat the more general case of signals given by an arbitrary linear combination of the inputs. We consider the dynamic shortterm or sequential memory defined as the capacity to reconstruct the signal $z(t)$ from the network state at a later time $t+\tau$ using a linear readout, $\sum_{i=1}^{K} w_{i} x_{i}(t+\tau)$, where $K \leq N$ is the number of readout neurons. The reconstruction capacity as a function of the time lag $\tau$ yields the memory curve $m(\tau)=1-\epsilon(\tau)$ [33], where $\epsilon(\tau)$ is the minimal relative mean-squared error between readout and signal. Alternatively, this measure quantifies the fidelity by which a sequence of past inputs can be reconstructed from the current network activity.

For optimal readout weights $w_{i}$ that minimize $\epsilon(\tau)$, the memory curve is given by $[33,55]$ 
$m(\tau)=\frac{\langle\boldsymbol{x}(t+\tau) z(t)\rangle^{\mathrm{T}}\left\langle\boldsymbol{x}(t) \boldsymbol{x}(t)^{\mathrm{T}}\right\rangle^{-1}\langle\boldsymbol{x}(t+\tau) z(t)\rangle}{\left\langle z(t)^{2}\right\rangle}$.

Here, the vector $\boldsymbol{x}(t)$ contains only the states of the $K$ readout neurons. We follow the approach by Toyoizumi and Abbott [9] and neglect the off-diagonal entries in $\left\langle\boldsymbol{x} \boldsymbol{x}^{\mathrm{T}}\right\rangle$, which is justifiable for a sparse readout with $K \ll N$. Additionally, for large $N$, the diagonal entries $\left\langle x_{i}^{2}\right\rangle$ are given by their mean-field value $c_{0}$, which is identical for all units. Determining the memory curve (27) then amounts to computing the sum of squared correlation functions $\sum_{i}\left\langle x_{i}(t+\tau) z(t)\right\rangle^{2}$ between the signal and the network activity, which we obtain by a replica calculation (Appendix G). The technique to perform a replica calculation, as it is known from disordered systems [34], is closely tied to the field-theoretical formulation chosen here. The key idea is to express the correlation functions $\left\langle x_{i} z\right\rangle$ as a sum of response functions $\left\langle x_{i} \tilde{x}_{j}\right\rangle$; this is possible because $z$ is Gaussian distributed. The calculation is similar to the derivation of the Schrödinger equation (Appendix C) with the difference, however, that the two replicas receive independent realizations of the inputs. The memory curve follows from a differential equation for the correlation between the two systems and is measured in units of the readout ratio $K / N(\mathrm{G} 22)$ :

$$
m(\tau)=\frac{2 \sigma^{2}}{c_{0}} \mathrm{e}^{-2 \tau} I_{0}\left[2 g\left\langle\phi^{\prime}(x)\right\rangle \tau\right] \Theta(\tau) d \tau,
$$

with the modified Bessel function of the first kind $I_{0}$ and the Heaviside step function $\Theta(\tau)$. The memory curve has two contributions (G22): memory due to the collective network dynamics and local memory due to the leaky integration of the single units. The latter effect is trivial and is reflected in the initial steep falloff as $\exp (-2 \tau)$ of the memory curves with time lag $\tau$, independent of the coupling strength [Fig. 6(a)]. Its decay time is half the time constant of the neurons, which is set to unity in Eq. (1). With increasing coupling strength, the variance $c_{0}$ increases, so the memory curve (28) decreases at zero time lag. For time lags that are large compared to the single-unit time constant, the network contribution to the memory dominates. A nonvanishing memory capacity for longer time lags is therefore only the result of the reverberation of the input through the network interaction. The analytical results are in excellent agreement with direct simulations [Figs. 6(a) and 6(b)].

We isolate the interesting network memory by subtracting the single-unit contribution:

$$
m_{\text {net }}(\tau)=m(\tau)-\frac{2 \sigma^{2}}{c_{0}} \mathrm{e}^{-2 \tau} \Theta(\tau) d \tau
$$

This quantity is particularly important in situations where the readout does not have access to the neurons receiving the signal. The network memory curve vanishes for the
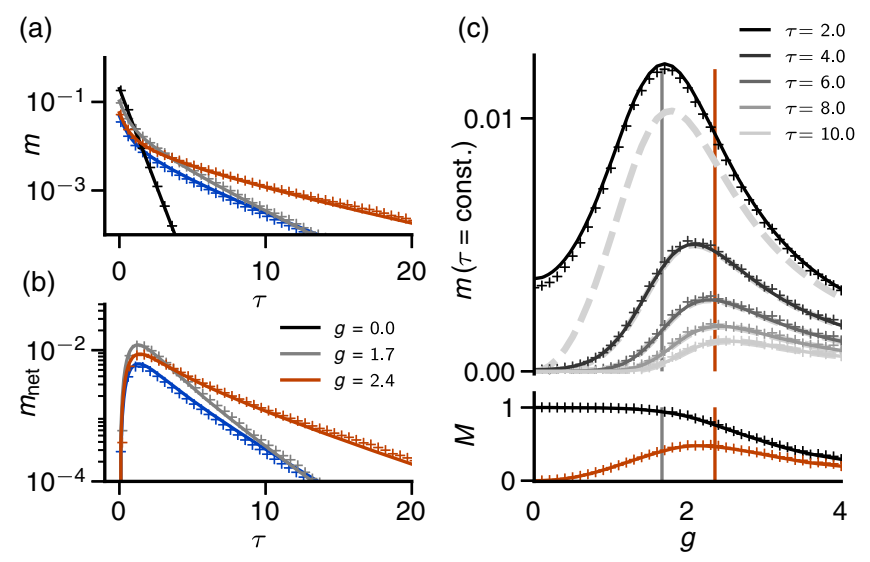

FIG. 6. Sequential memory. Mean-field prediction (solid curves) and simulation results (crosses). (a) Memory (28) as a function of time lag $\tau$ between signal and readout for different coupling strengths encoded in color [legend in panel (b)] for $\sigma=$ 1 and additionally for colored background noise with $\tau_{n}=2$ for $g=2.4$ and $\sigma=1$ (blue). (b) Corresponding network contribution (29) to memory. (c) Upper part of vertical axis: Memory at different time lags $\tau$ as a function of coupling strength $g$. Network contribution to memory shown as dashed thick light-gray curves, which coincide with total memory curves for $\tau \geq 4$. The vertical gray line marks local instability (18), and the vertical red line marks the transition to chaos [cf. Fig. 3(b)]. Lower part of vertical axis: Memory capacity $M$ (black), Eq. (30), and network contribution to memory capacity, $M_{\text {net }}$ (red).

uncoupled case. We compare the performance at two different coupling strengths: $g_{\text {nec }}$, defined by $\rho=$ $g_{\text {nec }} \sqrt{\left\langle\left[\phi^{\prime}(x)\right]^{2}\right\rangle}=1$ and corresponding to the onset of the local instability, and $g_{c}$, marking the onset of chaos [see Fig. 3(b)]. For short time lags, here for $\tau<4$, the network memory curve is larger for $g_{\text {nec }}$, while for longer time lags, it is larger for $g_{c}$ because of a slower decay of the memory curve. This behavior is confirmed by the memory curve as a function of $g$, shown for different time lags [Fig. 6(c), upper panel]. For $\tau \geq 4$, the memory capacity $m$ is entirely given by $m_{\text {net }}$, which is in line with the fast decay of the singleunit contribution. Moreover, while for small time lags the memory is maximal around $g_{\text {nec }}$, for larger time lags it peaks near $g_{\mathrm{c}}$, indicating that the intermediate, expansive, nonchaotic regime supports storage of the input.

The total memory capacity is defined as the integral over the memory curve:

$$
M=\int_{0}^{\infty} m(\tau)=\frac{\sigma^{2}}{c_{0}} \frac{1}{\sqrt{1-g^{2}\left\langle\phi^{\prime}(x)\right\rangle^{2}}}=\frac{\sigma^{2}}{c_{0}} \tau_{\infty},
$$

where the result follows directly from the Laplace transform of the Bessel function. Here, $\tau_{\infty}$ is the asymptotic decay time constant of the autocorrelation function, as defined above. Correspondingly, the total network memory capacity is defined as $M_{\text {net }}=\int m_{\text {net }}=M-\sigma^{2} / c_{0}$. Usually, the memory capacity is bounded by the number of neurons 
$N[33,55]$. The signal $z$ in our situation, however, can be seen as one out of $N$ independent inputs, and $m(\tau)$ is its corresponding memory curve. The expressions are therefore independent of $N$ [56], and the memory capacity satisfies $M \leq 1$. While the memory capacity decreases in the chaotic regime, the network memory peaks within the expansive, nonchaotic regime [Fig. 6(b), lower panel].

So far, we have considered the memory capacity at a fixed amplitude $\sigma$ of the input. In the following, we investigate the memory capacity over the whole phase diagram. The total memory capacity shows a steep falloff directly above the onset of chaos [Fig. 7(a)]. This is expected because the variance $c_{0}$ increases as the network dynamics becomes more chaotic. The contour lines of the memory capacity are nearly parallel to the transition criterion, the curve with a vanishing Lyapunov exponent. This observation closely links the transition to chaos to the
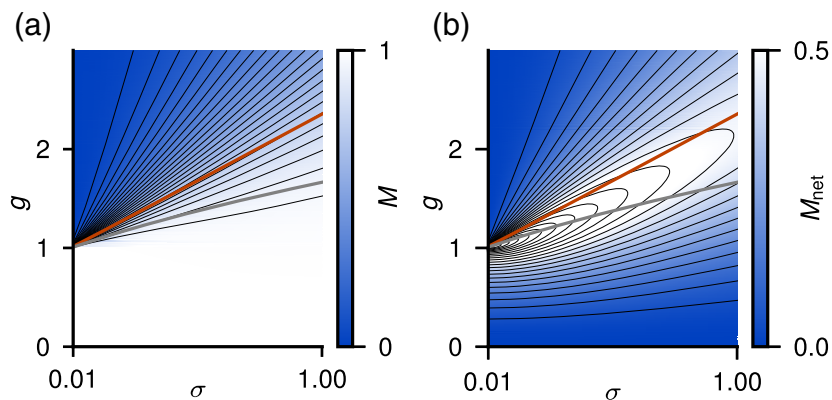

(c)
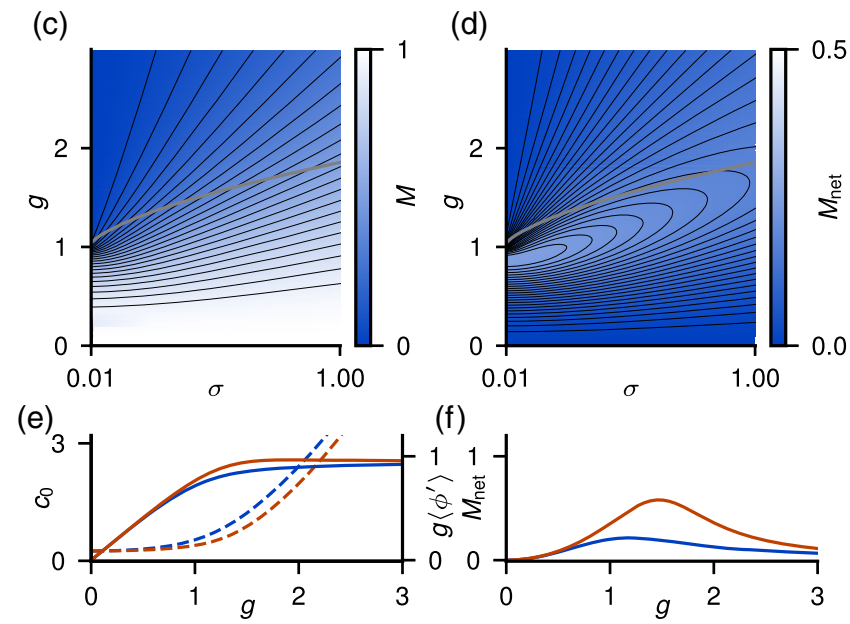

(f)

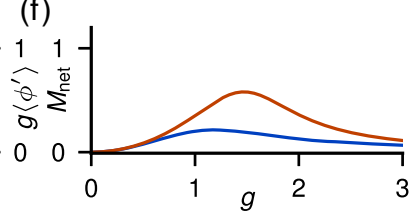

FIG. 7. Memory capacity in different phases of the network dynamics. (a) Total memory capacity (30) encoded in color. We show the transition curve (20) between regular and chaotic dynamics (red) and the necessary condition (18) of local instability (gray) as in Fig. 3. Contour lines of memory are shown in black. (b) Same as panel (a) for the network contribution to memory capacity. Panels (c) and (d) are the same as panels (a) and (b) but for quenched background noise. (e) Selfconsistent $c_{0}$ (dashed curve) and $g\left\langle\phi^{\prime}\right\rangle$ (solid curve) for whitenoise input (red) and quenched-noise input (blue) for $\sigma_{\mathrm{q}}=\sigma=0.5$. (f) Network contribution to memory capacity $M_{\text {net }}$ for white-noise input (red) and quenched-noise input (blue). memory capacity: The direction in the phase diagram in which the system most quickly enters the chaotic regime is accompanied by the steepest decline of memory. The contour lines of the network memory capacity show a ridge running through the expansive, nonchaotic regime [Fig. 7(b)] confirming the results found above: The memory is optimal in the dynamical regime of local instability and asymptotic stability. Moreover, the network capacity has a substantial contribution to the total memory capacity of about $50 \%$.

The optimal network memory in the hitherto unreported regime between $g_{\text {nec }}$ and $g_{\mathrm{c}}$ can be understood in an intuitive manner. Two conditions must be met for good memory. First, individual units must be susceptible to the signal; the susceptibility equals the noise-averaged slope $\left\langle\phi^{\prime}(x)\right\rangle$ of the transfer function. Second, the signal must propagate effectively through the network, requiring a sufficiently strong coupling $g$. These two requirements are reflected in the monotonic increase of the memory curve (28) with the effective slope $g\left\langle\phi^{\prime}\right\rangle$, independent of the time lag $\tau$. Increasing the coupling strength $g$, however, elevates internally generated fluctuations as well. The corresponding increase of the variance $c_{0}$ [Fig. 7(e)] has a twofold effect on the memory capacity. First, it decreases $\left\langle\phi^{\prime}\right\rangle$, so $g\left\langle\phi^{\prime}\right\rangle$ assumes a maximum [Fig. 7(e)], which is also reflected by the maximum of the decay time $\tau_{\infty}$ of the autocorrelation function [Fig. 3(c)]. Second, it reduces the signal-to-noise ratio of the readout, the prefactor $\sigma^{2} / c_{0}$ in Eqs. (28) and (30). The interplay of these two effects determines the location of the optimal memory within the phase diagram.

To further relate optimal memory to the dynamical behavior and to demonstrate the robustness of our results, we close this section by considering colored background noise with correlation time $\tau_{\mathrm{n}}$ as in Sec. V. So far, the memory task was to reconstruct a white-noise signal $z(t)$ on the background of the remaining white-noise inputs $\xi_{i}(t)-z(t)$. Since a signal with temporal correlations contains memory on its own, we keep the signal $z(t)$ as a white noise with amplitude $2 \sigma^{2} / N$, while the independent colored Gaussian background inputs $\xi_{i}(t)$ with autocorrelation function (D1) act as noise. This setup allows a direct comparison of the memory capacity in the driven network to the case of white background noise. In the uncoupled network, $g=0$, the colored background inputs lead to a variance $c_{0}=\sigma^{2}$ that is independent of the correlation time $\tau_{\mathrm{n}}$; the variance then increases with $g$ (see Sec. V). Hence, the input signal-to-noise ratio is $1 / N$, and the variance $c_{0}$ is predominantly determined by the background.

We find that the analytically derived memory curves (28) and (29) also hold for colored background noise because the autocorrelation function of the background does not enter the derivation of Eq. (G12). This finding is confirmed by simulations [Fig. 6(a)]. Indeed, the correlations of the background noise affect the memory only by changing the 
variance $c_{0}$. The qualitative behavior can be understood by comparing the peaks of the network memory within the phase diagrams for the two limiting cases of quenched and white-noise background input (Fig. 7).

For quenched background input, the network memory capacity peaks below the transition to local instability, which in this case also marks the transition to chaos [Fig. 7(c)]. To understand the emergence of the peak, we plot the decisive quantities $c_{0}$ and $g\left\langle\phi^{\prime}\right\rangle$ as a function of $g$ in Fig. 7(e); the corresponding network memory is shown in Fig. 7(f). The peak emerges at the point where $g\left\langle\phi^{\prime}\right\rangle$ begins to saturate, while the variance $c_{0}$ increases significantly. Now, compare this to the case of white-noise input: A corresponding increase of $c_{0}$ takes place only at larger values of $g$, and thus $g\left\langle\phi^{\prime}\right\rangle$ assumes higher values. The latter effect combined with the appearance of $c_{0}$ in the denominator of the memory leads to a substantial increase in the peak height as well as a shift of the maximum to higher coupling values [Fig. 7(f)]. Furthermore, the decrease in $c_{0}$, by Eq. (18), shifts the line of local instability to lower values of $g$ [compare Fig. 7(b) and 7(d)]. In combination, these effects switch the relative positions of the local stability line and the peak of the memory when going from quenched to white noise. Finally, due to the dynamic chaos suppression, the transition to chaos is located at even higher values of $g$, so the optimal memory is located in the expansive, nonchaotic regime.

\section{DISCUSSION}

Here, we present a completely solvable random network model that allows us to investigate the effect of timevarying input on the transition to chaos and informationprocessing capabilities. Adding time-varying stochastic drive to the seminal model by Sompolinsky et al. [1] yields a stochastic continuous-time dynamical system. Contrary to the original model [1], here we reformulate the stochastic differential equations as a field theory [25-28]. This formal step allows us to develop the dynamic mean-field theory by standard tools: a saddle-point approximation of the auxiliary-field generating functional $[17,30,36]$. As in the original model, this procedure reduces the interacting system to the dynamics of a single unit. The self-consistent solution of the effective equation yields a standard physics problem: The autocorrelation function of a typical unit is given by the motion of a classical particle in a potential. We find that the amplitude of the input corresponds to the initial kinetic energy of the particle.

Using the field-theoretical formulation, we then perform a replica calculation to determine the maximum Lyapunov exponent; the problem formally reduces to finding the ground-state energy of a single-particle quantum mechanical problem. The transition to chaos appears at the point where the ground-state energy changes sign, which allows us to obtain a closed-form condition relating the coupling strength and the input amplitude at the transition. We find a simple hallmark of the transition in the single-unit activity: At the transition point, the variance of the recurrent input to a single unit equals the variance of its own activity. Correspondingly, the autocorrelation function at zero time lag changes its curvature from convex to concave at the transition point. These features can readily be measured in most physical systems. The assessment of chaos by these passive observations, in particular, does not require a perturbation of the system.

The transition criterion allows us to map out the phase diagram spanned by the coupling strength and the input amplitude. It shows that stochastic drive shifts the transition to chaos to significantly larger coupling strengths than predicted by time-local linear stability analysis. The transition in the stochastic system is thus qualitatively different from the transition in the autonomous system, where loss of local stability and transition to chaos are equivalent $[1,21]$. The discrepancy of these two measures in the driven system is explained by a dynamic effect: The decrease of the maximum Lyapunov exponent is related to the sharpening of the autocorrelation function by the stochastic input. The displacement between local instability and transition to chaos leads to an intermediate regime that is absent in discrete-time networks of nonleaky units [23]. This hitherto unreported dynamical regime combines locally expansive dynamics with asymptotic stability.

The seminal works $[1,21]$ have established a tight link between the fields of random matrix theory and autonomous neural networks with random couplings: Deterministic chaos emerges if the spectral radius of the coupling matrix exceeds unity. In contrast, we find in stochastically driven networks that the spectral radius only yields a necessary condition for a positive Lyapunov exponent; it determines the minimum of the quantum mechanical potential whose ground-state energy relates to the Lyapunov exponent. The presented closed-form relation between input strength, the statistics of the random matrix, and the onset of chaos, Eq. (20), generalizes the well-known link to nonautonomous stochastic dynamics.

It is controversially discussed whether the instability of deterministic rate dynamics explains a transition to chaos in networks of spiking neurons [57-59]. It was argued that such a transition is absent in spiking models because the correlation time does not peak at the point where the corresponding deterministic rate dynamics becomes unstable $[43,58,59]$. For the analysis of oscillations [60] and correlations $[54,61]$ in these networks, the irregular spiking activity of the neurons can be approximated by effective stochastic rate equations, whereby the realization of the spikes is represented by an explicit source of noise. In this setting, the white-noise input in Eq. (1) can be interpreted as such spiking noise, explicitly investigated in Ref. [42]. For weak noise, one may neglect its impact on the location of the transition. In this limit, noise suppresses the divergence of the correlation time [42]. Moreover, the 
authors obtained scaling laws for the decay time and amplitude of the autocorrelation, indicative of a secondorder phase transition. Our work is not bound to small noise amplitudes, and it suggests that a diverging timescale in spiking networks does not occur at the instability for two reasons. First, we have shown that, in a stochastic system, the transition to chaos is not predicted by local instability. If a diverging timescale at the transition existed, it would not occur at the instability but at a larger coupling strength. But the presented analysis shows that the decay time of the autocorrelation function does not even peak at the transition to chaos but rather in the chaotic regime. While these results strictly only hold for the rate dynamics considered here, they still strongly suggest the absence of a diverging timescale in networks of spiking neurons. Indeed, the absence of a diverging timescale has been observed in the simulation of spiking neurons [58] as well as in an iterative approach solving for the self-consistent autocorrelation function $[62,63]$.

A complementary view on the transition to chaos in autonomous systems has been given by Wainrib and Touboul [64], showing that the number of fixed points of the dynamics, the topological complexity of the system, diverges at the transition in tight relation to the Lyapunov exponent, which measures the dynamical complexity. It is intriguing to ask how this view extends to the stochastic systems considered here. Investigating topological features of the fixed points of the effective action instead of the fixed points of the actual action may allow for addressing this question, drawing on the analogy between stochastic systems and their effective deterministic counterparts, which is exposed by the field-theoretical formulation presented here.

To assess whether this richer dynamics found in the driven network has functional consequences, we investigate sequential memory [33]. The obtained closed-form expression for the network memory capacity exhibits a peak within the expansive, nonchaotic regime. We identify two mechanisms whose partly antagonistic interplay causes optimal memory: local amplification of the stimulus and intrinsically generated noise. Local instability of the network ensures sensitivity to the external input, so on short timescales, the incoming signal is amplified and can therefore be read out more reliably. But larger coupling also increases networkintrinsic fluctuations, which, in turn, reduce the susceptibility as well as the signal-to-noise ratio of the readout. Therefore, it is plausible that the optimal memory appears at a point where local amplification of the external input is large enough, but intrinsic chaoticity is still limited.

Sequential memory has been studied in a discrete-time neural network model [9], which receives a single, weak, external input. In contrast, here we investigate the memory of a single signal in the presence of multiple simultaneous inputs with arbitrary amplitude $\sigma$. Without additional observation noise, Toyoizumi and Abbott [9] find that sequential memory does not possess a maximum; it is constant and optimal for subcritical coupling values $0<$ $g<1$ and falls off in the chaotic regime $g>1$ due to intrinsically generated fluctuations. Perfect reconstruction in the nonchaotic regime is possible because the one-step delayed activity is a direct linear function of the input. In our setting, the single neuron memory has a similar effect [Fig. 7(a)]. In the discrete-time system without additional input noise, optimal memory close to the transition arises only in the presence of observation noise [9]. In the chaotic phase, memory falls off more slowly than in the direction of regular dynamics, so fine-tuning is not needed if the network dynamics is slightly chaotic. The authors mention that the situation changes qualitatively in the presence of a small additional noise in the input: The signal-to-noise ratio then stays finite and peaks slightly below the transition to chaos.

In the continuous-time model studied here, we do not consider observation noise, but the remaining inputs act in a similar manner as the input noise mentioned in Ref. [9]. The network component of the memory or, equivalently, the memory for longer delay times $\tau$, also shows nonmonotonic behavior. For small signal amplitude $\sigma$, our analytical results show that memory is optimal right below the transition to chaos and steeply falls off above [Fig. 7(b)]; this is in line with the observation in the discrete-time setting with input noise [9]. For larger $\sigma$, the falloff is weaker in the chaotic regime, qualitatively more similar to the situation with observation noise.

A negative maximum Lyapunov exponent for nonautonomous dynamics indicates the echo state property, the reliability of the network response to input [65]. We could indeed show, for the analytically tractable model here, that memory capacity quickly declines in the chaotic regime due to intrinsically generated chaotic fluctuations. Echo state networks show long temporal memory near the edge of chaos [66-69]. Typically, these networks operate in discrete time, and thus, the onset of chaos is directly linked to the spectral radius of the Jacobian. This relation is used in the design of these systems, exploiting the fact that a spectral radius close to local instability ensures long memory times. Here, we show, for the driven and continuous-time system, that the edge of chaos and local instability are two different concepts and that memory capacity is a third, distinct measure: Memory is not optimal at either of the two other criteria but rather in between. In particular, our analytical results for the memory capacity can be used to determine the optimal coupling strength for a given input amplitude.

The finding that the edge of chaos does not necessarily coincide with the optimality of the measures of computation, as reported here, qualitatively alters the edge-of-chaos hypothesis leading to implications for methods that tune networks towards the edge of criticality with the goal to optimize performance [70]: The fundamental assumption of an equivalence of these a priori different measures is true for some discrete-time network dynamics [9]. But it 
certainly fails for nonautonomous networks operating in continuous time, as we show here. Moreover, the divergence of the timescale of the autocorrelation function in the autonomous setting [Fig. 3(c)] is indicative of critical slowing down [71] and could therefore point to a continuous phase transition. This phenomenon, however, vanishes in the nonautonomous case; the nature of the transition may hence be different. The presence of a continuous phase transition is an often-made second assumption to identify optimal network parameters $[70,72]$.

Recently, an algorithm was proposed to train a random network as given by Eq. (1) to produce a wide range of activity patterns [8]. Such learning shows the best performance if the random network without input is initially in the chaotic state. It has been argued that such networks have a large dynamic range and are able to produce a wide variety of outputs. In the training phase, the input to the network needs to suppress chaos so that learning converges. The procedure therefore requires the choice of an initial coupling that is large enough to ensure chaos but not too large so that the input can suppress chaos. Our quantitative criterion for the transition easily enables a proper choice of parameters and facilitates the design, control, and understanding of functional networks.

In this work, we have considered the memory of the input signal. An important task of the brain, however, is not only to maintain the input but also to perform nonlinear transformations on it. We expect the locally unstable but globally stable dynamics to be beneficial for such a task: The expansive behavior can project the input into a highdimensional space, which is crucial for nonlinear computations or discrimination tasks [67]. Thus, this dynamical regime not only provides memory but might serve as a basis for more complex computations.

To show the generic effect of input, we added a timevarying drive to the seminal model by Sompolinsky et al. [1]. Even though the original model makes some simplifying assumptions, such as the all-to-all Gaussian connectivity and a sigmoidal odd transfer function, the transition to chaos is qualitatively the same as in networks with biologically more realistic parameters $[20,42,43]$. To focus on the new physics arising in nonautonomous systems, here we start from the simplest but nontrivial and yet application-relevant model. Subsequently, we show that the mechanism is robust with regard to more realistic connectivity with nonvanishing mean and also for non-negative transfer functions, as they arise, for example, in rate model abstractions of spiking neurons. Preliminary results indicate that the same mechanism also persists in networks of populations of spiking neurons. Here, however, a suitable definition of rate chaos still needs to be found for a more quantitative advancement.

Our work predicts that time-varying input to the network modulates the distance to the transition to chaos, and it elucidates the corresponding mechanisms that control the transition. Consequently, we expect that the ongoing dynamics moves further away from the transition to chaos in periods in which the fluctuating drive to a network is elevated, thus potentially altering the processing capabilities along with the dimensionality of the intrinsic dynamics [73].

The reformulated derivation of the dynamic mean-field theory using established methods from field theory $[17,30,36,41,74]$ here allows us to find the explicit form of sequential memory by a replica calculation. In general, the presented formulation opens the study of recurrent neural networks to the rich and powerful set of fieldtheoretical methods developed in other branches of physics. This language leads to straightforward extension of our results in various directions. Among these directions are more biologically realistic settings, such as sparse connectivity respecting Dale's law [75] or multiple populations. The over-representation of bidirectional connections [76] can also be treated in this framework [77]. The extension to multiple populations would enable the study of the interesting case in which the population receiving the signal is separated from the readout population. Such a situation would most likely emerge in the cortex, where the input population of local microcircuits typically differs from the output population.

More generally, the stability of complex dynamical systems plays an important role in various other fields of physics, biology, and technology. Examples include oscillator networks [78], disordered soft-spin models [16], power grids [79], food webs [80], and gene-regulatory networks [81]. Presenting exact results for a prototypical and solvable model, this work contributes to the understanding of chaos and signal propagation in such highdimensional systems.

\section{ACKNOWLEDGMENTS}

We thank Rainer Engelken, Raoul-Martin Memmesheimer, and Tom Tetzlaff for helpful discussions. This work was partially supported by Helmholtz Young Investigator's Group VH-NG-1028, Helmholtz Portfolio Theme SMHB, Jülich Aachen Research Alliance (JARA). This project received funding from the European Union's Horizon 2020 Research and Innovation Programme under Grant Agreement No. 720270 . S. G. was partially supported by the German Federal Ministry of Education and Research (BMBF) through the Bernstein Network (01GQ1501 and 01GQ1710). J. S. was partially supported by the Fraunhofer Cluster of Excellence "Cognitive Internet Technologies".

\section{APPENDIX A: DERIVATION OF MEAN-FIELD EQUATION}

The generating functional $Z[\mathbf{I}](\mathbf{J})$ in Eq. (2) is properly normalized independent of the realization of $\mathbf{J}$. This property allows us to follow Ref. [28] and to introduce the disorder-averaged generating functional 


$$
\bar{Z}[\mathbf{l}]=\langle Z[\mathbf{l}](\mathbf{J})\rangle_{\mathbf{J}}=\int \Pi_{i j} d J_{i j} \mathcal{N}\left(0, \frac{g^{2}}{N}, J_{i j}\right) Z[\mathbf{I}](\mathbf{J}) .
$$

The coupling term $\exp \left(-\sum_{i \neq j} J_{i j} \tilde{x}_{i}^{\mathrm{T}} \phi\left(x_{j}\right)\right)$ in Eq. (2) over unit indices $i, j$, and the random weights $J_{i j}$ appear linear in the exponent. Thus, we can separately integrate over the independently and identically distributed $J_{i j}, i \neq j$, by completing the square, and we obtain

$$
\begin{aligned}
& \int d J_{i j} \mathcal{N}\left(0, \frac{g^{2}}{N}, J_{i j}\right) \exp \left(-J_{i j} \tilde{x}_{i}^{\mathrm{T}} \phi\left(x_{j}\right)\right) \\
& =\exp \left(\frac{g^{2}}{2 N}\left(\tilde{x}_{i}^{\mathrm{T}} \phi\left(x_{j}\right)\right)^{2}\right) .
\end{aligned}
$$

We reorganize the resulting sum in the exponent of the coupling term as

$$
\begin{aligned}
& \frac{g^{2}}{2 N} \sum_{i \neq j}\left(\int \tilde{x}_{i}(t) \phi\left(x_{j}(t)\right) d t\right)^{2} \\
& =\frac{g^{2}}{2 N} \sum_{i \neq j} \iint \tilde{x}_{i}(t) \phi\left(x_{j}(t)\right) \tilde{x}_{i}\left(t^{\prime}\right) \phi\left(x_{j}\left(t^{\prime}\right)\right) d t d t^{\prime} \\
& =\frac{1}{2} \sum_{i} \iint \tilde{x}_{i}(t) \tilde{x}_{i}\left(t^{\prime}\right)\left(\frac{g^{2}}{N} \sum_{j} \phi\left(x_{j}(t)\right) \phi\left(x_{j}\left(t^{\prime}\right)\right)\right) d t d t^{\prime} \\
& \quad-\frac{1}{2} \sum_{i} \iint \tilde{x}_{i}(t) \tilde{x}_{i}\left(t^{\prime}\right) \frac{g^{2}}{N} \phi\left(x_{i}(t)\right) \phi\left(x_{i}\left(t^{\prime}\right)\right) d t d t^{\prime},
\end{aligned}
$$

where we used $\left(\int f(t) d t\right)^{2}=\iint f(t) f\left(t^{\prime}\right) d t d t^{\prime}$ in the first step and $\sum_{i j} x_{i} y_{j}=\sum_{i} x_{i} \sum_{j} y_{j}$ in the second. The last line is the diagonal (self-coupling) to be skipped in the double sum. It is a correction of order $N^{-1}$ and will be neglected in the following. The disorder-averaged generating functional (A1) therefore takes the form

$$
\bar{Z}[\mathbf{l}]=\int \mathcal{D} \mathbf{x} \int \mathcal{D} \tilde{\mathbf{x}} \exp \left(S_{0}[\mathbf{x}, \tilde{\mathbf{x}}]+\mathbf{l}^{\mathrm{T}} \mathbf{x}+\frac{1}{2} \tilde{\mathbf{x}}^{\mathrm{T}} Q_{1} \tilde{\mathbf{x}}\right),
$$

where we extend our notation with $x^{\mathrm{T}} A y=\iint x(t) A\left(t, t^{\prime}\right) \times$ $y\left(t^{\prime}\right) d t d t^{\prime}$ to bilinear forms and define

$$
Q_{1}\left(t, t^{\prime}\right)=\frac{g^{2}}{N} \sum_{j} \phi\left(x_{j}(t)\right) \phi\left(x_{j}\left(t^{\prime}\right)\right) .
$$

The field $Q_{1}$ is an empirical average over $N$ contributions, which, by the law of large numbers and in the case of weak correlations, will converge to its expectation value for large $N$. This heuristic argument is validated in the following more formally: A saddle-point approximation leads to the replacement of $Q_{1}$ by its (self-consistent) expectation value. To this end, we first decouple the interaction term by inserting the Fourier representation of the Dirac delta functional:

$$
\begin{aligned}
\delta[ & \left.-\frac{N}{g^{2}} Q_{1}(t, s)+\phi(\mathbf{x}(t))^{\mathrm{T}} \phi(\mathbf{x}(s))\right] \\
& =\int \mathcal{D} Q_{2} \exp \left(-\frac{N}{g^{2}} Q_{1}^{\mathrm{T}} Q_{2}+\phi(\mathbf{x})^{\mathrm{T}} Q_{2} \phi(\mathbf{x})\right),
\end{aligned}
$$

where we further extend our notation with $Q_{1}^{\mathrm{T}} Q_{2}=\iint Q_{1}(t, s) Q_{2}(t, s) d t d s$ and $\phi(\mathbf{x}(t))^{\mathrm{T}} \phi(\mathbf{x}(s))=$ $\sum_{i=1}^{N} \phi\left(x_{i}(t)\right) \phi\left(x_{i}(s)\right)$. We note that the conjugate field $Q_{2}$ is purely imaginary. We hence rewrite Eq. (A4) as

$$
\begin{aligned}
\bar{Z}[j, \tilde{j}]= & \int \mathcal{D} Q_{1} \int \mathcal{D} Q_{2} \\
& \times \exp \left(-\frac{N}{g^{2}} Q_{1}^{\mathrm{T}} Q_{2}+N \ln \Omega\left[Q_{1}, Q_{2}\right]\right. \\
& \left.+j^{\mathrm{T}} Q_{1}+\tilde{j}^{\mathrm{T}} Q_{2}\right), \\
\Omega\left[Q_{1}, Q_{2}\right]= & \int \mathcal{D} x \int \mathcal{D} \tilde{x} \\
& \times \exp \left(S_{0}[x, \tilde{x}]+\frac{1}{2} \tilde{x}^{\mathrm{T}} Q_{1} \tilde{x}+\phi(x)^{\mathrm{T}} Q_{2} \phi(x)\right),
\end{aligned}
$$

where we introduce source terms $j, \tilde{j}$ for the auxiliary fields and drop the original source terms $\mathbf{I}^{\mathrm{T}} \mathbf{x}$. The integral measures $\mathcal{D} Q_{1,2}$ must be defined suitably. By writing $N \ln \Omega\left[Q_{1}, Q_{2}\right]$, we use the fact that the auxiliary fields couple only to sums of fields $\sum_{i} \phi^{2}\left(x_{i}\right)$ and $\sum_{i} \tilde{x}_{i}^{2}$, so the generating functional for the fields $\mathbf{x}$ and $\tilde{\mathbf{x}}$ factorizes into a product of $N$ identical factors $\Omega\left[Q_{1}, Q_{2}\right]$.

The remaining problem can be considered as a field theory for the auxiliary fields $Q_{1}$ and $Q_{2}$. The form (A7) clearly exposes the $N$ dependence of the action for these latter fields: It is of the form $\int d Q \exp (N f(Q))$, which, for large $N$, suggests a saddle-point approximation. This approximation neglects fluctuations in the auxiliary fields and hence sets them equal to their expectation value; this point is the dominant contribution to the probability mass. To obtain the saddle-point equations, we consider the Legendre-Fenchel transform of $\ln \bar{Z}$ as

$$
\Gamma\left[q_{1}, q_{2}\right]=\sup _{j, \tilde{j}}\left\{j^{\mathrm{T}} q_{1}+\tilde{j}^{\mathrm{T}} q_{2}-\ln \bar{Z}[j, \tilde{j}]\right\},
$$

which is called the vertex-generating functional or effective action [37,82]. It holds that $\delta \Gamma / \delta q_{1}=j$ and $\delta \Gamma / \delta q_{2}=\tilde{j}$, the so-called equations of state. The leading-order mean-field or tree-level approximation amounts to the approximation $\Gamma\left[q_{1}, q_{2}\right] \simeq-S\left[q_{1}, q_{2}\right]$, where $S\left[Q_{1}, Q_{2}\right]=$ $-\left(N / g^{2}\right) Q_{1}^{\mathrm{T}} Q_{2}+N \ln \Omega\left[Q_{1}, Q_{2}\right]$ is the action for the 
auxiliary fields $Q_{1}$ and $Q_{2}$. We insert this tree-level approximation into the equations of state and further set $j=\tilde{j}=0$ since the source fields have no physical meaning and thus must vanish. We get the saddle-point equations

$$
\begin{aligned}
0 & =\frac{\delta S\left[Q_{1}, Q_{2}\right]}{\delta Q_{\{1,2\}}} \\
& =\frac{\delta}{\delta Q_{\{1,2\}}}\left(-\frac{N}{g^{2}} Q_{1}^{\mathrm{T}} Q_{2}+N \ln \Omega\left[Q_{1}, Q_{2}\right]\right),
\end{aligned}
$$

from which we obtain a pair of equations

$$
\begin{aligned}
0 & =-\frac{N}{g^{2}} Q_{1}^{*}(t, s)+\left.\frac{N}{\Omega} \frac{\delta \Omega\left[Q_{1}, Q_{2}\right]}{\delta Q_{2}(t, s)}\right|_{Q^{*}} \\
\leftrightarrow Q_{1}^{*}(t, s) & =g^{2}\langle\phi(x(t)) \phi(x(s))\rangle_{Q^{*}}=g^{2} C_{\phi(x) \phi(x)}(t, s), \\
0 & =-\frac{N}{g^{2}} Q_{2}^{*}(t, s)+\left.\frac{N}{\Omega} \frac{\delta \Omega\left[Q_{1}, Q_{2}\right]}{\delta Q_{1}(t, s)}\right|_{Q^{*}} \\
\leftrightarrow Q_{2}^{*}(t, s) & =\frac{g^{2}}{2}\langle\tilde{x}(t) \tilde{x}(s)\rangle_{Q^{*}}=0,
\end{aligned}
$$

where we define the average autocorrelation function $C_{\phi(x) \phi(x)}(t, s)$ of the nonlinearly transformed activity of the units. The second saddle point $Q_{2}^{*}=0$ vanishes because the field was introduced to represent a Dirac delta constraint in the Fourier domain. One can show that, consequently, $\int \mathcal{D} Q \exp \left(S\left[Q_{1}, Q_{2}\right]\right) Q_{2}=0$, which is the true mean value $Q_{2}^{*}=\left\langle Q_{2}\right\rangle=0$.

Here, $\langle 0\rangle_{Q^{*}}$ denotes the expectation value with respect to realizations of $x$ evaluated at the saddle point $Q^{*}$. The expectation value must be computed self-consistently since the values of the saddle points, by Eq. (A7), influence the statistics of the fields $\mathbf{x}$, which in turn determines the function $Q_{1}^{*}$ by Eq. (A10). Inserting the saddle-point solution into the generating functional (A7), we get Eq. (4):

$$
\bar{Z}^{*} \propto \int \mathcal{D} x \int \mathcal{D} \tilde{x} \exp \left(S_{0}[x, \tilde{x}]+\frac{g^{2}}{2} \tilde{x}^{\mathrm{T}} C_{\phi(x) \phi(x)} \tilde{x}\right) .
$$

The action has the important property that it decomposes into a sum of actions for individual, noninteracting units which are driven by a field with common, self-consistently determined statistics, characterized by the second cumulant $C_{\phi(x) \phi(x)}$. Prior to the saddle-point approximation (A7), the fluctuations in the field $Q_{1}$ are common to all of the single units, effectively coupling them. The saddle-point approximation replaces the fluctuating field $Q_{1}$ by its mean (A10), which reduces the network to $N$ noninteracting units, or, equivalently, a single-unit system. The second term in Eq. (4) represents a Gaussian noise with a correlation function $g^{2} C_{\phi(x) \phi(x)}(t, s)$. Physically, it corresponds to the fluctuating input each unit receives from the $N-1$ other units. Its autocorrelation function is given by the summed autocorrelation functions of the output activities $\phi\left(x_{i}(t)\right)$ weighted by $g^{2} N^{-1}$, which incorporates the Gaussian distribution of the couplings.

The interpretation of the noise can be appreciated by explicitly considering the moment-generating functional of a Gaussian noise with a given autocorrelation function $C(t, s)$, which leads to the cumulant-generating functional $\ln Z_{\eta}[\tilde{x}]$ that appears in the exponent of Eq. (4) and has the form

$$
\ln Z_{\eta}[-\tilde{x}]=\ln \left\langle\exp \left(-\tilde{x}^{T} \eta\right)\right\rangle=\frac{1}{2} \tilde{x}^{\mathrm{T}} C \tilde{x} .
$$

Note that the only nonvanishing cumulant of the effective noise is the second cumulant; the cumulant-generating functional is quadratic in $\tilde{x}$. This means that the effective noise is Gaussian and only couples pairs of time points according to the correlation function.

\section{APPENDIX B: STATIONARY PROCESS}

We rewrite Eq. (5) as

$$
\left(\partial_{t}+1\right) x(t)=\tilde{\eta}(t),
$$

where we combine the two independent Gaussian processes $\eta(t)$ and $\xi(t)$ appearing in Eq. (5) into $\tilde{\eta}(t)$. We then multiply Eq. (B1) for time points $t$ and $s$ and take the expectation value over realizations of the noise $\tilde{\eta}$ on both sides, which leads to

$\left(\partial_{t}+1\right)\left(\partial_{s}+1\right) C_{x x}(t, s)=g^{2} C_{\phi(x) \phi(x)}(t, s)+2 \sigma^{2} \delta(t-s)$,

where we define the autocorrelation function of the activities $C_{x x}(t, s)=\langle x(t) x(s)\rangle$. We are now interested in the stationary statistics $C_{x x}(t, s)=c(t-s)$ of the system. The inhomogeneity in Eq. (B2) is then also time-translation invariant; $C_{\phi(x) \phi(x)}(t, s)$ is only a function of $\tau=t-s$. Therefore, the differential operator $\left(\partial_{t}+1\right)\left(\partial_{s}+1\right) c(t-s)$, with $\tau=t-s$, simplifies to $\left(-\partial_{\tau}^{2}+1\right) c(\tau)$, and we get

$$
\left(-\partial_{\tau}^{2}+1\right) c(\tau)=g^{2} C_{\phi(x) \phi(x)}(t+\tau, t)+2 \sigma^{2} \delta(\tau),
$$

given as Eq. (7) in the main text.

\section{APPENDIX C: REPLICA CALCULATION FOR THE MAXIMUM LYAPUNOV EXPONENT}

We start from the generating functional for the pair of systems (13) and perform the average over realizations of the coupling matrix $\mathbf{J}$, as in Eq. (A2). We therefore need to evaluate the Gaussian integral 


$$
\int d J_{i j} \mathcal{N}\left(0, \frac{g^{2}}{N}, J_{i j}\right) \exp \left(-J_{i j} \sum_{\alpha=1}^{2} \tilde{x}_{i}^{\alpha \mathrm{T}} \phi\left(x_{j}^{\alpha}\right)\right)=\exp \left(\frac{g^{2}}{2 N} \sum_{\alpha=1}^{2}\left[\tilde{x}_{i}^{\alpha \mathrm{T}} \phi\left(x_{j}^{\alpha}\right)\right]^{2}+\frac{g^{2}}{N}\left[\tilde{x}_{i}^{1 \mathrm{~T}} \phi\left(x_{j}^{1}\right)\right]\left[\tilde{x}_{i}^{2 \mathrm{~T}} \phi\left(x_{j}^{2}\right)\right]\right) .
$$

The first exponential factor only includes variables of a single subsystem and is identical to the term appearing in Eq. (A2). The second exponential factor is a coupling term between the two systems arising from the identical matrix $\mathbf{J}$ in the two replicas in each realization that enters the expectation value. We treat the former terms as before and concentrate on the mixed coupling term here. Analogous to Eq. (A3), the exponent of the mixed coupling term can be rewritten as

$$
\frac{g^{2}}{N} \sum_{i \neq j}\left[\tilde{x}_{i}^{1 \mathrm{~T}} \phi\left(x_{j}^{1}\right)\right]\left[\tilde{x}_{i}^{2 \mathrm{~T}} \phi\left(x_{j}^{2}\right)\right]=\iint \sum_{i} \tilde{x}_{i}^{1}(t) \tilde{x}_{i}^{2}(s) \frac{g^{2}}{N} \sum_{j} \phi\left(x_{j}^{1}(t)\right) \phi\left(x_{j}^{2}(s)\right) d t d s,
$$

where we include the self-coupling term $i=j$, which is again a subleading correction of order $N^{-1}$.

We now follow the steps in Appendix A and introduce three pairs of auxiliary variables. The pairs $Q_{1}^{\alpha}, Q_{2}^{\alpha}$ are defined as before in Eqs. (A5) and (A6), but for each subsystem, and the pair $T_{1}, T_{2}$ decouples the mixed term (C2) by defining

$$
T_{1}(t, s)=\frac{g^{2}}{N} \sum_{j} \phi\left(x_{j}^{1}(t)\right) \phi\left(x_{j}^{2}(s)\right) .
$$

Taken together, we can therefore rewrite the generating functional (13) averaged over the couplings as

$$
\begin{aligned}
\bar{Z}=\langle Z(\mathbf{J})\rangle_{\mathbf{J}}= & \Pi_{\alpha=1}^{2}\left\{\int \mathcal{D} Q_{1}^{\alpha} \int \mathcal{D} Q_{2}^{\alpha}\right\} \int \mathcal{D} T_{1} \int \mathcal{D} T_{2} \exp \left(S\left[\left\{Q_{1}^{\alpha}, Q_{2}^{\alpha}\right\}_{\alpha \in\{1,2\}}, T_{1}, T_{2}\right]\right), \\
S\left[\left\{Q_{1}^{\alpha}, Q_{2}^{\alpha}\right\}_{\alpha \in\{1,2\}}, T_{1}, T_{2}\right]= & -\sum_{\alpha=1}^{2} \frac{N}{g^{2}} Q_{1}^{\alpha \mathrm{T}} Q_{2}^{\alpha}-\frac{N}{g^{2}} T_{1}^{\mathrm{T}} T_{2}+N \ln \Omega^{12}\left[\left\{Q_{1}^{\alpha}, Q_{2}^{\alpha}\right\}_{\alpha \in\{1,2\}}, T_{1}, T_{2}\right], \\
\Omega^{12}\left[\left\{Q_{1}^{\alpha}, Q_{2}^{\alpha}\right\}_{\alpha \in\{1,2\}}, T_{1}, T_{2}\right]= & \Pi_{\alpha=1}^{2}\left\{\int \mathcal{D} x^{\alpha} \int \mathcal{D} \tilde{x}^{\alpha} \exp \left(S_{0}\left[x^{\alpha}, \tilde{x}^{\alpha}\right]+\frac{1}{2} \tilde{x}^{\alpha \mathrm{T}} Q_{1}^{\alpha} \tilde{x}^{\alpha}+\phi\left(x^{\alpha}\right)^{\mathrm{T}} Q_{2}^{\alpha} \phi\left(x^{\alpha}\right)\right)\right\} \\
& \times \exp \left(\tilde{x}^{\mathrm{T}}\left(T_{1}+2 \sigma^{2}\right) \tilde{x}^{2}+\phi\left(x^{1}\right)^{\mathrm{T}} T_{2} \phi\left(x^{2}\right)\right),
\end{aligned}
$$

where the generating functional factorizes into a product of $2 N$ identical factors $\Omega^{12}$.

Analogously to Appendix A, we can introduce sources for the auxiliary fields $Q_{1}^{\alpha}, Q_{2}^{\alpha}, T_{1}, T_{2}$. Then, the equations of state are obtained from the vertex-generating functional $\Gamma$ as before, which, in the tree-level approximation, is given by $\Gamma=-S$ and, for vanishing sources, leads to the saddlepoint equations $\delta S / \delta Q_{1,2}^{\alpha}=\delta S / \delta T_{1,2}=0$. From the latter, we obtain the set of equations

$$
\begin{aligned}
Q_{1}^{\alpha *}(t, s) & =g^{2} \frac{1}{\Omega^{12}} \frac{\delta \Omega^{12}}{\delta Q_{2}^{\alpha}(t, s)} \\
& =g^{2}\left\langle\phi\left(x^{\alpha}(t)\right) \phi\left(x^{\alpha}(s)\right)\right\rangle_{Q^{*}, T^{*}}, \\
Q_{2}^{\alpha *}(t, s) & =0, \\
T_{1}^{*}(t, s) & =g^{2} \frac{1}{\Omega^{12}} \frac{\delta \Omega^{12}}{\delta T_{2}(t, s)} \\
& =g^{2}\left\langle\phi\left(x^{1}(t)\right) \phi\left(x^{2}(s)\right)\right\rangle_{Q^{*}, T^{*}}, \\
T_{2}^{*}(t, s) & =0 .
\end{aligned}
$$

Therefore, the generating functional $\bar{Z}^{*}$ at the saddle point is

$$
\begin{aligned}
& \Pi_{\alpha=1}^{2}\left\{\int \mathcal{D} x^{\alpha} \int \mathcal{D} \tilde{x}^{\alpha} \exp \left(S_{0}\left[x^{\alpha}, \tilde{x}^{\alpha}\right]+\frac{1}{2} \tilde{x}^{\alpha \mathrm{T}} Q_{1}^{\alpha *} \tilde{x}^{\alpha}\right)\right\} \\
& \quad \times \exp \left(\tilde{x}^{1 \mathrm{~T}}\left(T_{1}^{*}+2 \sigma^{2}\right) \tilde{x}^{2}\right) .
\end{aligned}
$$

We make the following observations:

(1) The two subsystems $\alpha=1,2$ in the first line of Eq. (C5) have the same form as in Eq. (4). This consistency is expected because there is no physical coupling between the two systems. This implies that the marginal statistics of the activity in one system cannot be affected by the mere presence of the second. Hence, in particular, the saddle points $Q_{1,2}^{\alpha *}$ must be the same as in Eq. (4).

(2) If the term in the second line of Eq. (C5) was absent, the two systems would be independent. Two sources, however, contribute to correlations between the systems: the common Gaussian white noise that gives rise to the term proportional to $2 \sigma^{2}$ and the effective Gaussian noise due to a nonzero value of the auxiliary field $T_{1}^{*}(t, s)$. 
(3) Only products of pairs of fields appear in Eq. (C5), so $x^{\alpha}$ are Gaussian.

From Eqs. (C5) and (C4), we can read off the pair of effective dynamical equations (14) with self-consistent statistics (15).

\section{Derivation of the variational equation}

We multiply Eq. (14) with itself for equal or different values of $\alpha$ and take the expectation value on both sides. Thus, we get, for $\alpha, \beta \in\{1,2\}$,

$$
\begin{aligned}
& \left(\partial_{t}+1\right)\left(\partial_{s}+1\right) c^{\alpha \beta}(t, s) \\
& \quad=2 \sigma^{2} \delta(t-s)+g^{2} F_{\phi}\left(c^{\alpha \beta}(t, s), c^{\alpha \alpha}(t, t), c^{\beta \beta}(s, s)\right),
\end{aligned}
$$

where the function $F_{\phi}$ is defined as the expectation value

$$
F_{\phi}\left(c^{12}, c^{11}, c^{22}\right)=\left\langle\phi\left(x^{1}\right) \phi\left(x^{2}\right)\right\rangle
$$

for the centered bivariate Gaussian distribution

$$
\left(\begin{array}{l}
x^{1} \\
x^{2}
\end{array}\right) \sim \mathcal{N}_{2}\left(0,\left(\begin{array}{ll}
c^{11} & c^{12} \\
c^{12} & c^{22}
\end{array}\right)\right)
$$

First, we observe that the equations for the autocorrelation functions $c^{\alpha \alpha}(t, s)$ decouple and can each be solved separately, leading to the same equation (9) as before. This formal result could have been anticipated because the marginal statistics of each subsystem cannot be affected by the mere presence of the other system. Their stationary solutions

$$
c^{11}(t, s)=c^{22}(t, s)=c(t-s)
$$

then provide the "background" for the equation for the cross-correlation function between the two copies; they fix the second and third arguments of the function $F_{\phi}$ on the right-hand side of Eq. (C6). It remains to determine the equation of motion for $c^{12}(t, s)$.

We first determine the stationary solution $c^{12}(t, s)=$ $k(t-s)$. We see immediately from Eq. (C6) that $k(\tau)$ obeys the same equation of motion as $c(\tau)$, so $k(\tau)=c(\tau)$ is a solution. The distance (12) between replicas for this solution therefore vanishes; the dynamics in both replicas follows identical trajectories. Let us now study the stability of this solution. We hence need to expand $c^{12}$ around the stationary solution

$$
c^{12}(t, s)=c(t-s)+\epsilon k^{(1)}(t, s), \quad \epsilon \ll 1 .
$$

We expand the right-hand side of Eq. (C6) to linear order in $\epsilon$ using Price's theorem and Eq. (8):

$$
\begin{aligned}
F_{\phi}\left(c^{12}(t, s), c_{0}, c_{0}\right)= & f_{\phi}\left(c^{12},(t, s), c_{0}\right) \\
\simeq & f_{\phi}\left(c(t-s), c_{0}\right) \\
& +\epsilon f_{\phi^{\prime}}\left(c(t-s), c_{0}\right) k^{(1)}(t, s) .
\end{aligned}
$$

Inserting the expansion into Eq. (C6) and using the fact that $c(\tau)$ solves the zeroth-order equation, we get the equation of motion for the linear deflection (16). By Eq. (12), the deflection $k^{(1)}(t, s)$ determines the squared distance between the two subsystems as

$$
\begin{aligned}
d(t) & =\underbrace{c^{11}(t, t)}_{=c_{0}}+\underbrace{c^{22}(t, t)}_{=c_{0}} \underbrace{-c^{12}(t, t)-c^{21}(t, t)}_{=-2 c_{0}-2 \epsilon k^{(1)}(t, t)} \\
& =-2 \epsilon k^{(1)}(t, t) .
\end{aligned}
$$

\section{Schrödinger equation for the maximum Lyapunov exponent}

Here, we reformulate the equation for the variation of the cross-correlation (16) into a Schrödinger equation, as in the original work [see Eq. (10) in Ref. [1] ].

First, noting that $C_{\phi^{\prime} \phi^{\prime}}(t, s)=f_{\phi^{\prime}}\left(c(t-s), c_{0}\right)$ is timetranslation invariant, it is advantageous to introduce the coordinates $T=t+s$ and $\tau=t-s$ and write $k^{(1)}(t, s)$ as $k(T, \tau)$ with $k^{(1)}(t, s)=k(t+s, t-s)$. The differential operator $\left(\partial_{t}+1\right)\left(\partial_{s}+1\right)$ with the chain rule $\partial_{t} \rightarrow \partial_{T}+$ $\partial_{\tau}$ and $\partial_{s} \rightarrow \partial_{T}-\partial_{\tau}$ in the new coordinates is $\left(\partial_{T}+1\right)^{2}-\partial_{\tau}^{2}$. A separation ansatz $k(T, \tau)=\mathrm{e}^{\frac{1}{2} \kappa T} \psi(\tau)$ then yields the eigenvalue equation

$$
\left(\frac{\kappa}{2}+1\right)^{2} \psi(\tau)-\partial_{\tau}^{2} \psi(\tau)=g^{2} f_{\phi^{\prime}}\left(c(\tau), c_{0}\right) \psi(\tau)
$$

for the growth rates $\kappa$ of $d(t)=-2 \epsilon k^{(1)}(t, t)=-2 \epsilon k(2 t, 0)$. We can express the right-hand side by the second derivative of the potential $(\mathrm{C} 10)$ so that, with

$$
V^{\prime \prime}\left(c(\tau) ; c_{0}\right)=-1+g^{2} f_{\phi^{\prime}}\left(c(\tau), c_{0}\right),
$$

we get the time-independent Schrödinger equation

$$
\left(-\partial_{\tau}^{2}-V^{\prime \prime}\left(c(\tau) ; c_{0}\right)\right) \psi(\tau)=\underbrace{\left(1-\left(\frac{\kappa}{2}+1\right)^{2}\right)}_{=E} \psi(\tau),
$$

where the time lag $\tau$ plays the role of a spatial coordinate for the Schrödinger equation. The eigenvalues ("energies") $E_{n}$ determine the exponential growth rates $\kappa_{n}$ of the solutions $k(2 t, 0)=\mathrm{e}^{\kappa_{n} t} \psi_{n}(0)$ at $\tau=0$ with

$$
\kappa_{n}^{ \pm}=2\left(-1 \pm \sqrt{1-E_{n}}\right) .
$$


We can therefore determine the growth rate of the meansquare distance of the two subsystems by Eq. (9). The fastestgrowing mode of the distance is hence given by the ground-state energy $E_{0}$ and the plus in Eq. (12). The deflection between the two subsystems therefore grows with the rate

$$
\lambda_{\max }=\frac{1}{2} \kappa_{0}^{+}=-1+\sqrt{1-E_{0}},
$$

where the factor $1 / 2$ in the first line follows from $d$ being the squared distance.

\section{APPENDIX D: COLORED AND QUENCHED-NOISE INPUT}

\section{General case of colored Gaussian noise}

Here, we consider a network driven by $N$ independent colored Gaussian noise inputs $\xi_{i}(t)$ with zero mean and stationary covariance functions $\left\langle\xi_{i}(t+\tau) \xi_{j}(t)\right\rangle=$ $\delta_{i j} c_{\mathrm{cn}}(\tau)$. For concreteness, we use Ornstein-Uhlenbeck processes with autocorrelation function

$$
c_{\mathrm{cn}}(\tau)=\sigma^{2}\left(1+\frac{1}{\tau_{\mathrm{n}}}\right) \mathrm{e}^{-|\tau| / \tau_{\mathrm{n}}},
$$

where the correlation time $0<\tau_{\mathrm{n}} \leq \infty$ determines how fast the input varies in time. The limit $\tau_{\mathrm{n}} \rightarrow \infty$ leads to quenched-noise input, which is randomly Gaussian distributed but constant in time, while the limit $\tau_{\mathrm{n}} \rightarrow 0$ corresponds to Gaussian white-noise input as in Eq. (1). The cumulant-generating functional of the noise takes the form

$$
W_{\xi}[j]=\frac{1}{2} j^{\mathrm{T}} c_{\mathrm{cn}} j .
$$

It appears in the action of the disorder-averaged momentgenerating functional, analogous to Eq. (A4), as

$$
\begin{aligned}
\bar{Z}[\mathbf{l}]= & \langle Z[\mathbf{l}](\mathbf{J})\rangle_{\mathbf{J}} \\
= & \int \mathcal{D} \mathbf{x} \int \mathcal{D} \tilde{\mathbf{x}} \exp \left(\tilde{\mathbf{x}}^{\mathrm{T}}\left(\partial_{t}+1\right) \mathbf{x}+\frac{1}{2} \tilde{\mathbf{x}}^{\mathrm{T}} c_{\mathrm{cn}} \tilde{\mathbf{x}}+\mathbf{l}^{\mathrm{T}} \mathbf{x}\right) \\
& \times \exp \left(\frac{1}{2} \tilde{\mathbf{x}}^{\mathrm{T}} Q_{1} \tilde{\mathbf{x}}\right),
\end{aligned}
$$

where $Q_{1}$ is defined as before in Eq. (A5). Introducing a second auxiliary field $Q_{2}$ and performing the saddle-point approximation analogously to Appendix A, we obtain, from the stationary point for the auxiliary field $Q_{1}$, the differential equation for the stationary autocorrelation function $c(\tau)$ corresponding to Eq. (7):

$$
\left(-\partial_{\tau}^{2}+1\right) c(\tau)=g^{2} f_{\phi}\left(c(\tau), c_{0}\right)+c_{\mathrm{cn}}(\tau) .
$$

The factor $1+1 / \tau_{\mathrm{n}}$ in Eq. (D1) has been chosen such that, for $g=0$, the solution of Eq. (D3) yields the same variance $c_{0}=c(0)=\sigma^{2}$ for all correlation times $\tau_{\mathrm{n}}$ and is identical to the case of white-noise input with autocorrelation function $\left\langle\xi_{i}(t) \xi_{i}(s)\right\rangle=2 \sigma^{2} \delta(t-s)$.

Except in the case of quenched input, the second term in Eq. (D3) depends on $\tau$. If we rewrite this equation as a motion of a classical particle, this term corresponds to an additional, time-dependent force:

$$
\ddot{c}(\tau)=-V^{\prime}(c(\tau))-c_{\mathrm{cn}}(\tau),
$$

where the potential $V(c)=V\left(c ; c_{0}\right)$ is defined as in Eq. (10) and has to be determined self-consistently. Because of the time-dependent force, however, we cannot use energy conservation anymore to obtain $c_{0}$.

We may obtain a self-consistent solution numerically: Choosing an initial value $c(0)=c_{0} \geq \sigma^{2}$ arbitrarily, we integrate the differential equation (4) forward in time $\tau \geq 0$ based on the system

$$
\frac{d}{d t}\left(\begin{array}{c}
c \\
\dot{c}
\end{array}\right)=\left(\begin{array}{c}
\dot{c} \\
-V^{\prime}\left(c ; c_{0}\right)-c_{\mathrm{cn}}(\tau)
\end{array}\right),
$$

with the initial conditions $c(0)=c_{0}, \dot{c}(0)=0$. To determine the correct initial value $c_{0}$, we apply the shooting method to find the initial value $c_{0}$ at which $\lim _{\tau \rightarrow \infty} c(\tau)=0$. It leads to a self-consistent autocorrelation function $c(\tau)$, which monotonically decreases to zero.

\section{Quenched Gaussian noise}

The case of quenched Gaussian noise follows from the above by considering the limit $\tau_{n} \rightarrow \infty$ in Eq. (D1). We hence obtain from Eq. (D3) the differential equation for the autocorrelation function:

$$
\left(-\partial_{\tau}^{2}+1\right) c(\tau)=g^{2} f_{\phi}\left(c(\tau), c_{0}\right)+\sigma_{\mathrm{q}}^{2},
$$

where $\sigma_{\mathrm{q}}^{2}$ denotes the variance of the static quenched inputs with mean zero. Since the additional term in Eq. (D5) does not depend on $\tau$, we can include it into a potential and write

$$
\ddot{c}(\tau)=-V_{\mathrm{q}}^{\prime}(c(\tau))
$$

with the modified potential $V_{\mathrm{q}}\left(c ; c_{0}\right)=V\left(c ; c_{0}\right)+\sigma_{\mathrm{q}}^{2} c$. Again, the potential has to be determined self-consistently through the initial value $c(0)=c_{0}$. Without timedependent input, a constant autocorrelation function $c(\tau)=$ $c_{0}$ can be a solution; it is determined by $V_{\mathrm{q}}{ }^{\prime}\left(c_{0} ; c_{0}\right)=0$, which has a solution for all values of $g$. Depending on the coupling strength $g$, however, other time-dependent selfconsistent solutions exist as in the case without any input [1], which corresponds to $\sigma_{\mathrm{q}}^{2}=0$ here. 
We only consider a self-consistent autocorrelation function $c(\tau)$ that monotonically decays to an asymptotic value $c_{\infty}$, which is related to the variance of the quenched input $\sigma_{\mathrm{q}}^{2}$. In Sec. V, we describe how to construct this solution. Here, we briefly discuss its existence: Such a solution can only exist if the self-consistent potential $V_{\mathrm{q}}\left(c ; c_{0}\right)$ has both a maximum (at $c_{\infty}$ ) and a minimum (between $c_{\infty}$ and $c_{0}$ ) because the classical motion of $c(\tau)$ starts with zero kinetic energy, i.e., $\dot{c}(0)=0$. There exists a critical value $g_{\text {nec }}$ at which the second derivative at the endpoint of the potential $V_{\mathrm{q}}^{\prime \prime}\left(c_{0} ; c_{0}\right)=-1+g^{2}\left\langle\left[\phi^{\prime}(x)\right]^{2}\right\rangle$ changes sign from negative to positive; here, $c_{0}$ is determined for each $g$ by $V_{\mathrm{q}}{ }^{\prime}\left(c_{0} ; c_{0}\right)=0$. This implies that for $g>g_{\text {nec }}$, the selfconsistent potential $V_{\mathrm{q}}\left(c ; c_{0}\right)$ for a constant autocorrelation function $c(\tau)=c_{0}$ ends in a minimum and has an intermediate maximum. As a maximum followed by a minimum of the potential is necessary for the time-dependent, monotonically decaying, self-consistent, autocorrelation function, we conjecture that such a solution only exists for $g>g_{\text {nec }}$. This result agrees with the condition found for networks with internally generated quenched noise [20].

\section{Lyapunov exponent for colored and quenched-noise input}

The Lyapunov exponent for quenched or colored noise input can be obtained from the same Schrödinger eigenvalue equation (17) as in the white-noise case. This result follows from a replica calculation analogous to the whitenoise case (Appendix C), where the only difference is the form of the noise term. The saddle-point equation leads to a set of self-consistency equations for the correlations within and between the replicas, analogous to Eq. (C6). The explicit appearance of the noise term on the right-hand side vanishes in the equation for the distance, Eq. (12), after the linearization (8); hence, the correlation function of the noise enters only implicitly by determining the quantum potential (C10) in the Schrödinger equation (C11), where we need to insert the self-consistently determined autocorrelation function $c(\tau)$ obeying Eq. (D3) for colored noise or Eq. (5) for quenched noise.

\section{APPENDIX E: DYNAMIC MEAN-FIELD THEORY FOR NONVANISHING MEAN COUPLING}

In this Appendix, we treat the case of random couplings with nonzero mean. For concreteness, we assume here that the coupling weights are independently and identically Gaussian distributed according to

$$
J_{i j} \stackrel{\text { i.i.d. }}{\sim} \mathcal{N}\left(\frac{\bar{g}}{N}, \frac{g^{2}}{N}\right)
$$

Alternatively, we assume $J_{i j} \stackrel{\text { i.i.d. }}{\sim}\left(J_{0} / \sqrt{N}\right) \mathcal{B}(p)$, where $\mathcal{B}(p)$ denotes the Bernoulli distribution with success probability $p$. This assumption leads to a directed ErdósRényi network with connection probability $p$ and nonzero synaptic weights $J_{0} / \sqrt{N}$. We show below how the latter parameters $J_{0}, p$ determine the former ones $\bar{g}, g$ and that both networks are identical in the limit of large $N$. The network dynamics again follows Eq. (1). The moment-generating functional takes the same form as for the case of vanishing mean (2). Taking the disorder average, the calculation proceeds analogously to Eq. (A1). Introducing the abbreviation $y_{i j}=-\int \tilde{x}_{i}(t) \phi\left(x_{j}(t)\right) d t$, we may write the term in the action that is affected as

$$
\begin{aligned}
\left\langle\exp \left(-\tilde{x}^{\mathrm{T}} J \phi\right)\right\rangle_{J} & =\left\langle\exp \left(\sum_{i j} J_{i j} y_{i j}\right)\right\rangle_{J} \\
& =\exp \left(\frac{\bar{g}}{N} \sum_{i j} y_{i j}+\frac{1}{2} \frac{g^{2}}{N} \sum_{i j} y_{i j}^{2}\right) \\
& =\prod_{i} \exp \left(\tilde{x}_{i}^{\mathrm{T}} R_{1}+\frac{1}{2} \tilde{x}_{i}^{\mathrm{T}} Q_{1} \tilde{x}_{i}\right),
\end{aligned}
$$

where we introduce the auxiliary fields

$$
\begin{aligned}
R_{1}(t) & =\frac{\bar{g}}{N} \sum_{j} \phi\left(x_{j}(t)\right), \\
Q_{1}(t, s) & =\frac{g^{2}}{N} \sum_{j} \phi\left(x_{j}(t)\right) \phi\left(x_{j}(s)\right) .
\end{aligned}
$$

Analogously, the disorder average over an Erdős-Rényi network can be expressed by noting that its cumulantgenerating function for a single connection weight reads

$$
\begin{aligned}
\varphi_{J_{i j}}(y) & =\ln \left\langle\mathrm{e}^{y\left(J_{0} / \sqrt{N}\right) s}\right\rangle_{S \sim \mathcal{B}(p)} \\
& =\ln \left(p \mathrm{e}^{\left(J_{0} / \sqrt{N}\right) y}-1\right) \\
& =\frac{J_{0} p}{\sqrt{N}} y+\frac{p(1-p) J_{0}^{2}}{2 N} y^{2}+O\left(N^{-\frac{3}{2}}\right),
\end{aligned}
$$

where the first two cumulants $p\left(J_{0} / \sqrt{N}\right)$ and $p(1-$ $p)\left(J_{0}^{2} / N\right)$ appear as coefficients in the polynomial in $y$.

We therefore get the same form for the disorder-averaged action as Eq. (E2), but with different scalar factors in the definition of the auxiliary fields as $R_{1}(t)=\left[\left(J_{0} p\right) /\right.$ $\sqrt{N}] \sum_{j} \phi\left(x_{j}(t)\right) \quad$ and $\quad Q_{1}(t, s)=\left\{\left[p(1-p) J_{0}^{2}\right] / 2 N\right\} \times$ $\sum_{j} \phi\left(x_{j}(t)\right) \phi\left(x_{j}(s)\right)$. The terms that originate from cumulants of order higher than 2 are suppressed for large network size with at least $O\left(N^{-\frac{3}{2}}\right)$, so they do not contribute in the limit of large $N$. Comparing to Eq. (E3), we may hence treat the Erdős-Rényi network on the same footing as the network with Gaussian connectivity by identifying the parameters as 


$$
\begin{gathered}
\bar{g}=\sqrt{N} J_{0} p, \\
g^{2}=p(1-p) J_{0}^{2} .
\end{gathered}
$$

We now derive the mean-field equations for the system. The four-point coupling term involving $Q_{1}$ is handled in the same manner as before by introducing an auxiliary field $Q_{2}$ that serves to represent a functional delta as in Eq. (A6). Analogously, we perform a second HubbardStratonovich transform to write $\delta\left[-(N / \bar{g}) R_{1}+\sum_{i} \phi\left(x_{i}\right)\right] \propto$ $\int \mathcal{D} R_{2} \exp \left(-(N / \bar{g}) R_{2}^{\mathrm{T}} R_{1}+R_{2}^{\mathrm{T}} \sum_{i} \phi\left(x_{i}\right)\right)$ to obtain the disorder-averaged moment-generating functional

$\bar{Z}[k, \tilde{k}, j, \tilde{j}]=\iint\left\{\mathcal{D} R_{1,2}\right\} \iint\left\{\mathcal{D} Q_{1,2}\right\} \exp \left(-\frac{N}{\bar{g}} R_{1}^{\mathrm{T}} R_{2}-\frac{N}{g^{2}} Q_{1}^{\mathrm{T}} Q_{2}+N \ln \Omega\left[R_{1}, R_{2}, Q_{1}, Q_{2}\right]+k^{\mathrm{T}} R_{1}+\tilde{k}^{\mathrm{T}} R_{2}+j^{\mathrm{T}} Q_{1}+\tilde{j}^{\mathrm{T}} Q_{2}\right)$,

$$
\Omega\left[R_{1}, R_{2}, Q_{1}, Q_{2}\right]=\int \mathcal{D} x \int \mathcal{D} \tilde{x} \exp \left(S_{0}[x, \tilde{x}]-R_{1}^{\mathrm{T}} \tilde{x}+R_{2}^{\mathrm{T}} \phi(x)+\frac{1}{2} \tilde{x}^{\mathrm{T}} Q_{1} \tilde{x}+\phi(x)^{\mathrm{T}} Q_{2} \phi(x)\right)
$$

where again the generating functional factorizes into independent, identical single unit contributions $\Omega$, giving rise to the factor $N$ in front of $\ln \Omega$ in the first line.

To formally derive a consistent approximation, it is standard to define the vertex-generating functional or effective action as

$$
\begin{aligned}
\Gamma\left[r_{1}, r_{2}, q_{1}, q_{2}\right]= & \sup _{\{j, \tilde{j}, k, \tilde{k}\}}\left\{k^{\mathrm{T}} r_{1}+\tilde{k}^{T} r_{2}+j^{\mathrm{T}} q_{1}+\tilde{j}^{\mathrm{T}} q_{2}\right. \\
& -\ln Z[k, \tilde{k}, j, \tilde{j}]\} .
\end{aligned}
$$

The equations of state

$$
\frac{\delta \Gamma}{\delta\left\{r_{1}, r_{2}, q_{1}, q_{2}\right\}}=0
$$

solved for the auxiliary fields, then yield a coupled set of self-consistency equations for these fields in the absence of external sources.

In the lowest order, the tree-level approximation [37], the effective action $\Gamma$ is approximated by the negative action:

$$
\begin{aligned}
\Gamma\left[r_{1}, r_{r}, q_{1}, q_{2}\right] \simeq & -S\left[r_{1}, r_{2}, q_{1}, q_{2}\right], \\
S\left[r_{1}, r_{2}, q_{1}, q_{2}\right]= & -\frac{N}{\bar{g}} r_{1}^{\mathrm{T}} r_{2}-\frac{N}{g^{2}} q_{1}^{\mathrm{T}} q_{2} \\
& +N \ln \Omega\left[r_{1}, r_{2}, q_{1}, q_{2}\right] .
\end{aligned}
$$

The self-consistency equations (E6) for the auxiliary fields then take the explicit form

$$
\begin{aligned}
& 0=\frac{\delta \Gamma}{\delta r_{1}} \rightarrow R_{2}^{*}(t)=\bar{g}\langle\tilde{x}(t)\rangle_{\Omega}=0, \\
& 0=\frac{\delta \Gamma}{\delta r_{2}} \rightarrow R_{1}^{*}(t)=\bar{g}\langle\phi(x(t))\rangle_{\Omega}, \\
& 0=\frac{\delta \Gamma}{\delta q_{1}} \rightarrow Q_{2}^{*}(s, t)=\frac{g^{2}}{2}\langle\tilde{x}(s) \tilde{x}(t)\rangle_{\Omega}=0, \\
& 0=\frac{\delta \Gamma}{\delta q_{2}} \rightarrow Q_{1}^{*}(s, t)=g^{2}\langle\phi(x(s)) \phi(x(t))\rangle_{\Omega},
\end{aligned}
$$

where $\langle 0\rangle_{\Omega}$ is the expectation value with respect to the statistics of the fields $x$ and $\tilde{x}$ given by $\Omega$ in Eq. (E5), with the saddle-point values for the auxiliary fields inserted. The effective equation of motion follows from inserting the saddle-point values into the action as

$$
S_{0}[x, \tilde{x}]-R_{1}^{* \mathrm{~T}} \tilde{x}+\frac{1}{2} \tilde{x}^{\mathrm{T}} Q_{1}^{*} \tilde{x}
$$

which demonstrates that we get a mean input proportional to $R_{1}^{*}$ and Gaussian fluctuations with covariance $Q_{1}^{*}$, analogous to Appendix B:

$$
\begin{aligned}
\left(\partial_{t}+1\right) x(t) & =\bar{g}\langle\phi(t)\rangle+\eta(t), \\
\langle\eta(t) \eta(s)\rangle & =Q_{1}^{*}(t, s)+2 \sigma^{2} \delta(t-s),
\end{aligned}
$$

where $\eta(t)$ is again a Gaussian process with zero mean. Hence, the stationary solution for the mean value satisfies the nonlinear self-consistency equation

$$
\langle x\rangle=\bar{g}\langle\phi\rangle .
$$

Defining as $\delta x(t)=x(t)-\langle x\rangle$ the deflection from the mean, we get a differential equation for the stationary covariance function $c(\tau)=\langle\delta x(t+\tau) \delta x(t)\rangle$ [using the equation of motion (E10) with the mean $\langle x\rangle$ subtracted on both sides], 


$$
\left(-\partial_{\tau}^{2}+1\right) c(\tau)=g^{2} C_{\phi(x) \phi(x)}(t+\tau, t)+2 \sigma^{2} \delta(\tau),
$$

which is identical to Eq. (7).

The new term is therefore the mean input $\bar{g}\langle\phi\rangle$ from the network. We can obtain the joint solution of $\langle\phi\rangle$ and $C_{\phi \phi}$ from the coupled system of equations,

$$
\begin{aligned}
\langle\phi(x)\rangle & =\int D z \phi\left(\langle x\rangle+\sqrt{c_{0}} z\right), \\
C_{\phi \phi}(\tau) & =f_{\phi(\circ+\langle x\rangle)}\left(c_{0}, c(\tau)\right),
\end{aligned}
$$

where $f_{u}\left(c_{0}, c_{1}\right)$ is defined in Eq. (8) and $D z$ denotes the standard Gaussian measure. Here, we need to insert the function $\phi(\circ+\langle x\rangle)$ to capture the nonzero mean of $x$; the Gaussian fluctuating part is contained in the definition of $f$.

Note that for $\langle x\rangle \neq 0, C_{\phi \phi}(\tau)$ does not decay to 0 as $\tau \rightarrow \infty$, but it approaches a nonzero asymptotic value $C_{\phi \phi}(\infty)=f_{\phi(\circ+\langle x\rangle)}\left(c_{0}, c_{\infty}\right)$. This signifies that the disorder in the connectivity contributes to a distribution of mean activities across units in the population.

\section{APPENDIX F: LYAPUNOV EXPONENT FOR NONVANISHING MEAN COUPLING}

The calculation of the maximum Lyapunov exponent in the case of nonvanishing mean of the connections proceeds along the same line as in Appendix C. We therefore only highlight the steps in which the calculation differs from the former case.

As shown in Appendix E, Erdős-Rényi networks and networks with Gaussian connectivity lead to the same limiting equations for large $N$. So, without loss of generality, we keep the notation of Gaussian connectivity here. To assess the Lyapunov exponent, one needs to compute the disorder-average over a pair of systems (13). The only term affected takes the form

$$
\begin{aligned}
& \int d J_{i j} \mathcal{N}\left(\frac{\bar{g}^{2}}{N}, \frac{g^{2}}{N}, J_{i j}\right) \exp \left(-J_{i j} \sum_{\alpha=1}^{2} \tilde{x}_{i}^{\alpha \mathrm{T}} \phi\left(x_{j}^{\alpha}\right)\right) \\
& =\exp \left(-\frac{\bar{g}^{2}}{N} \sum_{\alpha=1}^{2} \tilde{x}_{i}^{\alpha \mathrm{T}} \phi\left(x_{j}^{\alpha}\right)\right) \\
& \quad \times \exp \left(\frac{g^{2}}{2 N} \sum_{\alpha=1}^{2}\left[\tilde{x}_{i}^{\alpha \mathrm{T}} \phi\left(x_{j}^{\alpha}\right)\right]^{2}\right) \\
& \quad \times \exp \left(\frac{g^{2}}{N}\left[\tilde{x}_{i}^{\mathrm{T}} \phi\left(x_{j}^{1}\right)\right]\left[\tilde{x}_{i}^{2 \mathrm{~T}} \phi\left(x_{j}^{2}\right)\right]\right),
\end{aligned}
$$

where the only difference from Eq. (C1) is the additional first factor on the right-hand side. The important property of this additional term is that it does not couple variables belonging to different replicas; hence, it does not contribute to the correlation between the two replicas. As we demonstrate below, the analysis of the transition to chaos therefore remains unchanged.

As a direct consequence, the corresponding saddle-point equations for the auxiliary variables $R_{1,2}^{\alpha}$ are identical to Eq. (E9) and hence read

$$
\begin{aligned}
& R_{2}^{* \alpha}(t)=\bar{g}\left\langle\tilde{x}^{\alpha}(t)\right\rangle_{\Omega}=0, \\
& R_{1}^{* \alpha}(t)=\bar{g}\left\langle\phi\left(x^{\alpha}(t)\right)\right\rangle_{\Omega} .
\end{aligned}
$$

Performing the same steps as in Appendix $\mathrm{C}$ leads to the same form of the saddle-point equations (C4) for the remaining fields, so the generating functional at the saddle point takes the form

$$
\bar{Z}^{*} \propto \iint \Pi_{\alpha=1}^{2} \mathcal{D} x^{\alpha} \mathcal{D} \tilde{x^{\alpha}} \exp \left(\sum_{\alpha=1}^{2} S_{0}\left[x^{\alpha}, \tilde{x}^{\alpha}\right]-R_{1}^{* \alpha \mathrm{T}} \tilde{x}_{\alpha}+\frac{1}{2} \tilde{x}^{\alpha \mathrm{T}} Q_{1}^{\alpha *} \tilde{x}^{\alpha}+\tilde{x}^{1 \mathrm{~T}}\left(T_{1}^{*}+2 \sigma^{2}\right) \tilde{x}^{2}\right)
$$

analogous to Eq. (C5). The only differences are the terms $-\sum_{\alpha=1}^{2} R_{1}^{* \alpha \mathrm{T}} \tilde{x}_{\alpha}$, which correspond to the mean input received by the neuron in each replicon. The corresponding set of effective equations can be read off in the same manner as before, yielding Eq. (24) given in the main text.

\section{APPENDIX G: MEMORY CURVE}

To evaluate Eq. (27), we need to determine the disorderaveraged sum of squared response functions

$$
\sum_{i=1}^{K} \overline{\left\langle x_{i}(t) z\left(t_{0}\right)\right\rangle^{2}}
$$

with $t_{0}=t-\tau$ and $K$ denoting the number of neurons connected to the readout, which we initially leave as a free parameter. Here, $\langle 0\rangle$ denotes the average over realizations of the inputs $\xi_{i}(t)$ (or, alternatively, over time), and the overbar is the average over realizations of the connectivity $\mathbf{J}$ as in Eq. (A1). Moreover, here we examine a more general signal $z(t)=\sum_{j=1}^{N} v_{j} \xi_{j}(t)$, a linear combination with weights $v_{i}$ of the inputs to different neurons.

We pick two points in time $t, s \geq t_{0}$ and define

$$
h^{K}(t, s)=\sum_{i=1}^{K} \overline{\left\langle x_{i}(t) z\left(t_{0}\right)\right\rangle\left\langle x_{i}(s) z\left(t_{0}\right)\right\rangle} .
$$

The measure of interest (G1) then follows for $t=s$. The key idea is to express the correlation function $\left\langle x_{i}(t) z\left(t_{0}\right)\right\rangle$ 
as a weighted sum of response functions $\left\langle x_{i}(t) \tilde{x}\left(t_{0}\right)\right\rangle$, which we show in the following. We introduce a scalar source term $k(t)$ for the signal $z(t)$ and average over the inputs. This yields the generating functional

$$
\begin{aligned}
Z[\mathbf{l}, k]= & \int \mathcal{D} \mathbf{x} \int \mathcal{D} \tilde{\mathbf{x}} \exp \left(S_{0}[\mathbf{x}, \tilde{\mathbf{x}}]-\tilde{\mathbf{x}}^{\mathrm{T}} \mathbf{J} \phi(\mathbf{x})\right) \\
& \times \exp \left(\mathbf{l}^{\mathrm{T}} \mathbf{x}-\sum_{i} 2 \sigma^{2} v_{i} k^{\mathrm{T}} \tilde{x}_{i}\right) .
\end{aligned}
$$

Evaluating the correlation leads to

$$
\begin{aligned}
\left\langle x_{i}(t) z\left(t_{0}\right)\right\rangle & =\left.\frac{\delta^{2} Z}{\delta l_{i}(t) \delta k\left(t_{0}\right)}\right|_{l_{i}=k=0} \\
& =-2 \sigma^{2} \sum_{j=1}^{N} v_{j}\left\langle x_{i}(t) \tilde{x}_{j}\left(t_{0}\right)\right\rangle .
\end{aligned}
$$

We now consider a pair of systems (replicas) similar to Appendix C, with the difference being that the two systems receive independent realizations of the inputs. We need two independent systems to express the product of the two correlation functions in Eq. (G1). By independence, the corresponding average factorizes

$$
\begin{aligned}
\left\langle x_{i}(t) z\left(t_{0}\right)\right\rangle\left\langle x_{i}(s) z\left(t_{0}\right)\right\rangle & =\left\langle x_{i}^{1}(t) z^{1}\left(t_{0}\right) x_{i}^{2}(s) z^{2}\left(t_{0}\right)\right\rangle \\
& =h_{i}\left(t, s, t_{0}\right),
\end{aligned}
$$

where the superscript denotes the replicon index as before. It is sufficient to introduce a single source term

$$
4 \sigma^{4} \sum_{j, l} v_{j} v_{l} \int d t \epsilon(t) \tilde{x}_{j}^{1}(t) \tilde{x}_{l}^{2}(t),
$$

with source $\epsilon(t)$, to the corresponding generating functional, which allows us to obtain $\left\langle x_{i}(t) z\left(t_{0}\right)\right\rangle$ with Eqs. (G4) and (G5) as

$$
\left\langle x_{i}(t) z\left(t_{0}\right)\right\rangle\left\langle x_{i}(s) z\left(t_{0}\right)\right\rangle=\left.\frac{\delta}{\delta l_{i}^{1}(t)} \frac{\delta}{\delta l_{i}^{2}(s)} \frac{\delta}{\delta \epsilon\left(t_{0}\right)} Z\right|_{l_{i}^{1}=l_{i}^{2}=\epsilon=0} .
$$

The additional source term (G6) has the physical interpretation of a common input with time-dependent variance $\epsilon(t)$ injected into a pair of units between the two replicas. The absence of quadratic terms $\left(\tilde{x}^{\alpha}\right)^{2}$ shows that this common input does not affect the marginal statistics of the two systems in isolation. This interpretation is only mentioned here for illustrative purposes; the derivation does not rely on it. Because of the weight $v_{j} v_{l}$ for different neuron pairs $j$, $l$, we keep the neuron index in the following.

To compute Eq. (G1), we need the disorder average of Eq. (G5), $\overline{h_{i}\left(t, s, t_{0}\right)}$. Similar to Appendix C 1, we proceed by deriving a differential equation for this function.
First, after averaging over the disorder, completely analogous to Appendix $\mathrm{C}$, we can read off effective equations for the single units,

$$
\left(\partial_{t}+1\right) x_{i}^{\alpha}(t)=\xi_{i}^{\alpha}(t)+\eta_{i}^{\alpha}(t)+\rho_{i}^{\alpha}(t),
$$

$\alpha \in\{1,2\}, i \in\{1, \ldots, N\}$, together with a set of selfconsistency equations for the correlations of the noises:

$$
\begin{aligned}
\left\langle\xi_{i}^{\alpha}(t) \xi_{j}^{\beta}(s)\right\rangle & =2 \sigma^{2} \delta_{\alpha \beta} \delta_{i j} \delta(t-s), \\
\left\langle\eta_{i}^{\alpha}(t) \eta_{j}^{\beta}(s)\right\rangle & =\frac{g^{2}}{N} \delta_{i j} \sum_{i}\left\langle\phi\left(x_{i}^{\alpha}(t)\right) \phi\left(x_{i}^{\beta}(s)\right)\right\rangle, \\
\left\langle\rho_{i}^{\alpha}(t) \rho_{j}^{\beta}(s)\right\rangle & =4 \sigma^{4}\left(1-\delta_{\alpha \beta}\right) v_{i} v_{j} \epsilon(t) \delta(t-s) .
\end{aligned}
$$

The first line in Eq. (G8) represents the independent noise between the systems, the second line the common connectivity, and the third line the common noise component we introduced in Eq. (G6) to express the squared response function (G2).

Second, we obtain $\overline{h_{i}\left(t, s, t_{0}\right)}$ by a functional derivative with respect to $l_{i}^{1}(t), l_{i}^{2}(s)$, and $\epsilon\left(t_{0}\right)$, which follows from the representation (G5). Writing the functional derivative with respect to $\epsilon$ explicitly as a limit, we can express it by the correlation between the pair of systems:

$$
\overline{h_{i}\left(t, s, t_{0}\right)}=\left.\lim _{l \rightarrow 0} \frac{1}{l}\left\langle x_{i}^{1}(t) x_{i}^{2}(s)\right\rangle\right|_{\epsilon=l \delta\left(\circ-t_{0}\right)},
$$

where we use that for $\epsilon=0$, the two systems are uncorrelated. We now combine the effective equations (G7) and (G9) to obtain a partial differential equation:

$$
\begin{aligned}
\left(\partial_{t}+1\right)\left(\partial_{s}+1\right) \overline{h_{i}\left(t, s, t_{0}\right)} \\
=\left.\frac{g^{2}}{N} \lim _{l \rightarrow 0} \frac{1}{l}\left[\sum_{j=1}^{N}\left\langle\phi\left(x_{j}^{1}(t)\right) \phi\left(x_{j}^{2}(s)\right)\right\rangle\right]\right|_{\epsilon=l \delta\left(\circ-t_{0}\right)} \\
\quad+4 \sigma^{4} v_{i}^{2} \delta(t-s) \delta\left(t-t_{0}\right) .
\end{aligned}
$$

Since we are interested in the limit $l \rightarrow 0$, we expand to linear order around the uncorrelated state:

$$
\begin{aligned}
\left\langle\phi\left(x_{i}^{1}(t)\right) \phi\left(x_{i}^{2}(s)\right)\right\rangle & =f_{\phi}\left(0, c_{0}\right)+\partial_{c} f_{\phi}\left(0, c_{0}\right) c_{i}^{12}(t, s) \\
& =\bar{\imath} \overline{\left\langle\phi^{\prime}\left(x_{i}\right)\right\rangle^{2}} \overline{h_{i}\left(t, s, t_{0}\right)},
\end{aligned}
$$

where the first term vanishes as $\overline{\langle\phi(x)}^{2}=0$. Inserting Eq. (G11) into Eq. (G10), we arrive at

$$
\begin{aligned}
\left(\partial_{t}\right. & +1)\left(\partial_{s}+1\right) \overline{h_{i}\left(t, s, t_{0}\right)} \\
& =\frac{g^{2}}{N} \overline{\left\langle\phi^{\prime}\left(x_{j}\right)\right\rangle^{2}} \sum_{j=1}^{N} \overline{h_{j}\left(t, s, t_{0}\right)}+4 \sigma^{4} v_{i}^{2} \delta\left(t-t_{0}\right) \delta\left(s-t_{0}\right) .
\end{aligned}
$$


Here, $\overline{\left\langle\phi^{\prime}\left(x_{j}\right)\right\rangle}$ is independent of $j$ because the expectation value is taken with respect to the disorder-averaged unperturbed system; thus, we use a representative unit $j$ as the index. Taking the sum over $i=1, \ldots, K$ yields

$$
\begin{aligned}
& \left(\partial_{t}+1\right)\left(\partial_{s}+1\right) h^{K}(t, s) \\
& \quad=g^{2}{\overline{\left\langle\phi^{\prime}\left(x_{j}\right)\right\rangle^{2}}}^{2} \frac{K}{N} h^{N}(t, s)+4 \sigma^{4}\left\|\mathbf{v}_{K}\right\|^{2} \delta\left(t-t_{0}\right) \delta\left(s-t_{0}\right)
\end{aligned}
$$

with $\left\|\mathbf{v}_{K}\right\|^{2}=\sum_{i=1}^{K} v_{i}^{2}$. For the complete sum of squared response functions, $h^{N}(t, s)$, the following closed linear partial differential equation holds:

$$
\begin{aligned}
& \left(\partial_{t}+1\right)\left(\partial_{s}+1\right) h^{N}(t, s) \\
& \quad=g^{2} \overline{\left\langle\phi^{\prime}\left(x_{j}\right)\right\rangle^{2}} h^{N}(t, s)+4 \sigma^{4} \delta\left(t-t_{0}\right) \delta\left(s-t_{0}\right),
\end{aligned}
$$

where we set $\left\|\mathbf{v}_{N}\right\|^{2}=\|\mathbf{v}\|^{2}=1$ without loss of generality. In the main text, we consider $\|\mathbf{v}\|^{2}=1 / N$, which only leads to an additional factor in the following and cancels in the formula for the memory curve. The solution to this equation describes the shape of the memory curve if the readout has access to the states of all neurons. To determine $h^{K}(t, s)$, we note that the difference $h^{K}(t, s)-$ $(K / N) h^{N}(t, s)$ is proportional to the solution of

$$
\left(\partial_{t}+1\right)\left(\partial_{s}+1\right) h^{(0)}(t, s)=\delta\left(t-t_{0}\right) \delta\left(s-t_{0}\right),
$$

which by direct integration yields

$$
h^{(0)}(t, s)=\mathrm{e}^{-\left(t-t_{0}\right)} \Theta\left(t-t_{0}\right) \mathrm{e}^{-\left(s-t_{0}\right)} \Theta\left(s-t_{0}\right) .
$$

Thus, $h^{K}(t, s)$ is given by

$$
\begin{aligned}
h^{K}(t, s) & =\frac{K}{N} h^{N}(t, s)+4 \sigma^{4}\left(\left\|\mathbf{v}_{K}\right\|^{2}-\frac{K}{N}\right) h^{(0)}(t, s) \\
& =4 \sigma^{4} \frac{K}{N} h^{(1)}(t, s)+4 \sigma^{4}\left(\left\|\mathbf{v}_{K}\right\|^{2}-\frac{K}{N}\right) h^{(0)}(t, s),
\end{aligned}
$$

where $h^{(1)}(t, s)$ solves

$$
\begin{aligned}
& \left(\partial_{t}+1\right)\left(\partial_{s}+1\right) h^{(1)}(t, s) \\
& \quad=a^{2} h^{(1)}(t, s)+\delta\left(t-t_{0}\right) \delta\left(s-t_{0}\right)
\end{aligned}
$$

with parameter $a^{2}=g^{2} \overline{\left\langle\phi^{\prime}\left(x_{j}\right)\right\rangle^{2}}=1-1 / \tau_{\infty}^{2}$. Here, $\tau_{\infty}$ is the asymptotic decay time of the autocorrelation function.

As in Appendix C 2, it is useful to change coordinates to $T=t+s-2 t_{0}$ and $\tau=t-s$. In these coordinates, Eq. (G17) takes the form

$$
\begin{gathered}
\left(\partial_{T}+1\right)^{2} h^{(1)}(T, \tau)-\partial_{\tau}^{2} h^{(1)}(T, \tau) \\
=a^{2} h^{(1)}(T, \tau)+2 \delta(T) \delta(\tau)
\end{gathered}
$$

and setting $h^{(1)}(T, \tau)=\mathrm{e}^{-T} u(T, \tau)$ simplifies the equation further to

$$
\partial_{T}^{2} u(T, \tau)-\partial_{\tau}^{2} u(T, \tau)=a^{2} u(T, \tau)+2 \delta(T) \delta(\tau),
$$

a Klein-Gordon wave equation with temporal coordinate $T$ and spatial coordinate $\tau$ (and negative squared mass $-a^{2}$ ). We are looking for the solution $u(T, \tau)$ in $T \geq 0, \tau \in \mathbb{R}$. To this end, we consider the temporal Laplace and the spatial Fourier transform of Eq. (G18). Fourier transformation in $\tau$ yields

$$
\partial_{T}^{2} \hat{u}(T, k)+\left(k^{2}-a^{2}\right) \hat{u}(T, k)=2 \delta(T),
$$

with $k \in \mathbb{R}$ and the Fourier representation

$$
u(T, \tau)=\frac{1}{2 \pi} \int_{-\infty}^{+\infty} e^{i k \tau} \hat{u}(T, k) d k .
$$

For each $k \in \mathbb{R}$, the Laplace transformation in $T$,

$$
\tilde{u}(p, k)=\int_{0}^{\infty} e^{-p T} \hat{u}(T, k) d T,
$$

of Eq. (G19) reads

$$
p^{2} \tilde{u}(p, k)-p \underbrace{\hat{u}(0, k)}_{=0}-\partial_{T} \underbrace{\hat{u}(0, k)}_{=0}+\left(k^{2}-a^{2}\right) \tilde{u}(p, k)=2 .
$$

Hence, in the Fourier-Laplace domain, we obtain

$$
\tilde{u}(p, k)=\frac{2}{p^{2}+k^{2}-a^{2}} .
$$

For the memory curve, we only need the solution $u(T, \tau)$ for $\tau=0$, the diagonal $s=t$ in the original coordinates. Setting $\tau=0$ in the Fourier representation gives the Laplace transform of $u(T, \tau=0)$ :

$$
\begin{aligned}
\tilde{u}(p)=\tilde{u}(p, \tau=0) & =\frac{1}{\pi} \int_{-\infty}^{+\infty} \frac{1}{k^{2}+p^{2}-a^{2}} d k \\
& =\frac{1}{\sqrt{p^{2}-a^{2}}},
\end{aligned}
$$

with $p \in \mathbb{C}$ such that $\operatorname{Re}\left(p^{2}\right)>a^{2}$. The function on the right is the Laplace transform of the modified Bessel function of the first kind $I_{0}(a T)$ [83]. Together with $h^{(1)}(T, \tau)=\mathrm{e}^{-T} u(T, \tau)$, we therefore obtain the shape of the memory curve as 


$$
h^{(1)}(T)=h^{(1)}(T, \tau=0)=\mathrm{e}^{-T} I_{0}(a T) \Theta(T) .
$$

Finally, using Eqs. (G15) and (G21) in Eq. (G16) gives the following explicit expression (setting $t_{0}=0$ ) for the sum of squared response functions:

$$
\begin{aligned}
h_{K}(t)= & h_{K}(t, t)=4 \sigma^{4} \frac{K}{N} \mathrm{e}^{-2 t} I_{0}(a 2 t) \Theta(t) \\
& +4 \sigma^{4}\left(\left\|\mathbf{v}_{K}\right\|^{2}-\frac{K}{N}\right) \mathrm{e}^{-2 t} \Theta(t) \\
= & 4 \sigma^{4} \frac{K}{N} \mathrm{e}^{-2 t}\left(I_{0}(a 2 t)-1\right) \Theta(t) \\
& +4 \sigma^{4}\left\|\mathbf{v}_{K}\right\|^{2} \mathrm{e}^{-2 t} \Theta(t) .
\end{aligned}
$$

In Eq. (G22), we split $h_{K}(t)$ into two contributions: a network contribution $h_{K}^{\text {net }}(t)$ proportional to $K / N$ with shape $\mathrm{e}^{-2 t}\left(I_{0}(a 2 t)-1\right) \Theta(t)$ and a local contribution proportional to $\left\|\mathbf{v}_{K}\right\|^{2}$ with shape $\mathrm{e}^{-2 t} \Theta(t)$. The latter is just the memory of the signal due to the leaky integration of the single units, while the former describes the memory due to the collective network dynamics; only this contribution is affected by the network coupling strength

We can evaluate Eq. (27) using Eq. (G22) with the choice $v_{i}=1 / N$, which leads to Eq. (28) and to the network memory (29).

[1] H. Sompolinsky, A. Crisanti, and H. J. Sommers, Chaos in Random Neural Networks, Phys. Rev. Lett. 61, 259 (1988).

[2] C. van Vreeswijk and H. Sompolinsky, Chaos in Neuronal Networks with Balanced Excitatory and Inhibitory Activity, Science 274, 1724 (1996).

[3] M. Monteforte and F. Wolf, Dynamical Entropy Production in Spiking Neuron Networks in the Balanced State, Phys. Rev. Lett. 105, 268104 (2010).

[4] G. Lajoie, K. K. Lin, and E. Shea-Brown, Chaos and Reliability in Balanced Spiking Networks with Temporal Drive, Phys. Rev. E 87, 052901 (2013).

[5] W. Maass, T. Natschläger, and H. Markram, Real-Time Computing without Stable States: A New Framework for Neural Computation Based on Perturbation, Neural Comput. 14, 2531 (2002).

[6] H. Jaeger and H. Haas, Harnessing Nonlinearity: Predicting Chaotic Systems and Saving Energy in Wireless Communication, Science 304, 78 (2004).

[7] R. Legenstein and W. Maass, Edge of Chaos and Prediction of Computational Performance for Neural Circuit Models, Neural Netw. 20, 323 (2007).

[8] D. Sussillo and L. F. Abbott, Generating Coherent Patterns of Activity from Chaotic Neural Networks, Neuron 63, 544 (2009).

[9] T. Toyoizumi and L. F. Abbott, Beyond the Edge of Chaos: Amplification and Temporal Integration by Recurrent Networks in the Chaotic Regime, Phys. Rev. E 84, 051908 (2011).
[10] O. Barak, D. Sussillo, R. Romo, M. Tsodyks, and L. F. Abbott, From Fixed Points to Chaos: Three Models of Delayed Discrimination, Prog. Neurobiol. 103, 214 (2013).

[11] K. Rajan, C. D. Harvey, and D. W. Tank, Recurrent Network Models of Sequence Generation and Memory, Neuron 90 , 128 (2016).

[12] N. Li, K. Daie, K. Svoboda, and S. Druckmann, Robust Neuronal Dynamics in Premotor Cortex During Motor Planning, Nature (London) 532, 459 (2016).

[13] J. D. Murray, A. Bernacchia, N. A. Roy, C. Constantinidis, R. Romo, and X.-J. Wang, Stable Population Coding for Working Memory Coexists with Heterogeneous Neural Dynamics in Prefrontal Cortex, Proc. Natl. Acad. Sci. U.S.A. 114, 394 (2016).

[14] R. Laje and D. V. Buonomano, Robust Timing and Motor Patterns by Taming Chaos in Recurrent Neural Networks, Nat. Neurosci. 16, 925 (2013).

[15] V. Mante, D. Sussillo, K. V. Shenoy, and W. T. Newsome, Context-dependent Computation by Recurrent Dynamics in Prefrontal Cortex, Nature (London) 503, 78 (2013).

[16] H. Sompolinsky and A. Zippelius, Dynamic Theory of the Spin-Glass Phase, Phys. Rev. Lett. 47, 359 (1981).

[17] H. Sompolinsky and A. Zippelius, Relaxational Dynamics of the Edwards-Anderson Model and the MeanField Theory of Spin-Glasses, Phys. Rev. B 25, 6860 (1982).

[18] K. Rajan, L. F. Abbott, and H. Sompolinsky, Stimulusdependent Suppression of Chaos in Recurrent Neural Networks, Phys. Rev. E 82, 011903 (2010).

[19] J. Aljadeff, M. Stern, and T. Sharpee, Transition to Chaos in Random Networks with Cell-Type-Specific Connectivity, Phys. Rev. Lett. 114, 088101 (2015).

[20] O. Harish and D. Hansel, Asynchronous Rate Chaos in Spiking Neuronal Circuits, PLoS Comput. Biol. 11, e1004266 (2015).

[21] H. Sommers, A. Crisanti, H. Sompolinsky, and Y. Stein, Spectrum of Large Random Asymmetric Matrices, Phys. Rev. Lett. 60, 1895 (1988).

[22] K. Rajan and L. F. Abbott, Eigenvalue Spectra of Random Matrices for Neural Networks, Phys. Rev. Lett. 97, 188104 (2006).

[23] L. Molgedey, J. Schuchhardt, and H. Schuster, Suppressing Chaos in Neural Networks by Noise, Phys. Rev. Lett. 69, 3717 (1992).

[24] C. Zhou and J. Kurths, Noise-induced Phase Synchronization and Synchronization Transitions in Chaotic Oscillators, Phys. Rev. Lett. 88, 230602 (2002).

[25] P. Martin, E. Siggia, and H. Rose, Statistical Dynamics of Classical Systems, Phys. Rev. A 8, 423 (1973).

[26] H.-K. Janssen, On a Lagrangean for Classical Field Dynamics and Renormalization Group Calculations of Dynamical Critical Properties, Z. Phys. B 23, 377 (1976).

[27] C. De Dominicis, Renormalization Techniques for Fields and Dynamics of Critical Phenomena, J. Phys. (Paris), Colloq. 37, C1 (1976).

[28] C. De Dominicis and L. Peliti, Field-Theory Renormalization and Critical Dynamics above $T_{c}$ : Helium, Antiferromagnets, and Liquid-Gas Systems, Phys. Rev. B 18, 353 (1978). 
[29] J. Schuecker, S. Goedeke, D. Dahmen, and M. Helias, Functional Methods for Disordered Neural Networks, arXiv:1605.06758.

[30] M. Moshe and J. Zinn-Justin, Quantum Field Theory in the Large N Limit: A Review, Phys. Rep. 385, 69 (2003).

[31] J.-P. Eckmann and D. Ruelle, Ergodic Theory of Chaos and Strange Attractors, Rev. Mod. Phys. 57, 617 (1985).

[32] B. Derrida and Y. Pomeau, Random Networks of Automata: A Simple Annealed Approximation, Europhys. Lett. 1, 45 (1986).

[33] H. Jaeger, Short Term Memory in Echo State Networks (GMD-Forschungszentrum Informationstechnik, Sankt Augustin, 2001), Vol. 5.

[34] K. Fischer and J. Hertz, Spin Glasses (Cambridge University Press, Cambridge, England, 1991).

[35] S.-I. Amari, Characteristics of Random Nets of Analog Neuron-like Elements, IEEE Trans. Syst. Man Cybernetics SMC-2, 643 (1972).

[36] A. Crisanti and H. Sompolinsky, Dynamics of Spin Systems with Randomly Asymmetric Bonds: Langevin Dynamics and a Spherical Model, Phys. Rev. A 36, 4922 (1987).

[37] J. Zinn-Justin, Quantum Field Theory and Critical Phenomena (Clarendon Press, Oxford, 1996).

[38] T. Cabana and J. Touboul, Large Deviations, Dynamics and Phase Transitions in Large Stochastic and Disordered Neural Networks, J. Stat. Phys. 153, 211 (2013).

[39] C. W. Gardiner, Handbook of Stochastic Methods for Physics, Chemistry and the Natural Sciences, 2nd ed., Springer Series in Synergetics No. 13 (Springer-Verlag, Berlin, 1985).

[40] A. Altland and B. Simons, Condensed Matter Field Theory (Cambridge University Press, Cambridge, England, 2010).

[41] C. Chow and M. Buice, Path Integral Methods for Stochastic Differential Equations, J. Math. Neurosci. 5, 8 (2015).

[42] J. Kadmon and H. Sompolinsky, Transition to Chaos in Random Neuronal Networks, Phys. Rev. X 5, 041030 (2015).

[43] F. Mastrogiuseppe and S. Ostojic, Intrinsically-Generated Fluctuating Activity in Excitatory-Inhibitory Networks, PLoS Comput. Biol. 13, e1005498 (2017).

[44] R. Price, A Useful Theorem for Nonlinear Devices Having Gaussian Inputs, IRE Trans. Inf. Theory 4, 69 (1958).

[45] A. Papoulis, Probability, Random Variables, and Stochastic Processes, 3rd ed. (McGraw-Hill, Boston, Massachusetts, 1991).

[46] The theory of random dynamical systems makes this more precise; a brief overview is given in Ref. [4].

[47] S. Kirkpatrick and D. Sherrington, Infinite-ranged Models of Spin-Glasses, Phys. Rev. B 17, 4384 (1978).

[48] J. Hahne, D. Dahmen, J. Schuecker, A. Frommer, M. Bolten, M. Helias, and M. Diesmann, Integration of Continuous-Time Dynamics in a Spiking Neural Network Simulator, Front. Neuroinformatics 11, 34 (2017).

[49] A. Peyser et al., NEST 2.14.0. Zenodo (2017), https://doi .org/10.5281/zenodo.882971.

[50] S. Ostojic and N. Brunel, From Spiking Neuron Models to Linear-Nonlinear Models, PLoS Comput. Biol. 7, e1001056 (2011).
[51] N. Fourcaud and N. Brunel, Dynamics of the Firing Probability of Noisy Integrate-and-Fire Neurons, Neural Comput. 14, 2057 (2002).

[52] N. Brunel, F. S. Chance, N. Fourcaud, and L. F. Abbott, Effects of Synaptic Noise and Filtering on the Frequency Response of Spiking Neurons, Phys. Rev. Lett. 86, 2186 (2001).

[53] B. Lindner and L. Schimansky-Geier, Transmission of Noise Coded versus Additive Signals through a Neuronal Ensemble, Phys. Rev. Lett. 86, 2934 (2001).

[54] M. Helias, T. Tetzlaff, and M. Diesmann, Echoes in Correlated Neural Systems, New J. Phys. 15, 023002 (2013).

[55] J. Dambre, D. Verstraeten, B. Schrauwen, and S. Massar, Information Processing Capacity of Dynamical Systems, Sci. Rep. 2, 514 (2012).

[56] M. Hermans and B. Schrauwen, Memory in Reservoirs for High Dimensional Input, The 2010 International Joint Conference on Neural Networks (IJCNN) (IEEE, New York, 2010), pp. 1-7, http://dx.doi.org/10.1109/ IJCNN.2010.5596884.

[57] S. Ostojic, Two Types of Asynchronous Activity in Networks of Excitatory and Inhibitory Spiking Neurons, Nat. Neurosci. 17, 594 (2014).

[58] R. Engelken, F. Farkhooi, D. Hansel, C. van Vreeswijk, and F. Wolf, A Reanalysis of "Two Types of Asynchronous Activity in Networks of Excitatory and Inhibitory Spiking Neurons", F1000Research 5, 2043 (2016).

[59] S. Ostojic, Response to Comment on "Two Types of Asynchronous Activity in Networks of Excitatory and Inhibitory Spiking Neurons," bioRxiv, 020354 (2015).

[60] N. Brunel and V. Hakim, Fast Global Oscillations in Networks of Integrate-and-Fire Neurons with Low Firing Rates, Neural Comput. 11, 1621 (1999).

[61] J. Trousdale, Y. Hu, E. Shea-Brown, and K. Josic, Impact of Network Structure and Cellular Response on Spike Time Correlations, PLoS Comput. Biol. 8, e1002408 (2012).

[62] B. Dummer, S. Wieland, and B. Lindner, Self-Consistent Determination of the Spike-Train Power Spectrum in a Neural Network with Sparse Connectivity, Front. Comput. Neurosci. 8, 104 (2014).

[63] S. Wieland, D. Bernardi, T. Schwalger, and B. Lindner, Slow Fluctuations in Recurrent Networks of Spiking Neurons, Phys. Rev. E 92, 040901 (2015).

[64] G. Wainrib and J. Touboul, Topological and Dynamical Complexity of Random Neural Networks, Phys. Rev. Lett. 110, 118101 (2013).

[65] G. Wainrib and M. N. Galtier, A Local Echo State Property through the Largest Lyapunov Exponent, Neural Netw. 76, 39 (2016).

[66] N. Bertschinger and T. Natschläger, Real-Time Computation at the Edge of Chaos in Recurrent Neural Networks, Neural Comput. 16, 1413 (2004).

[67] R. Legenstein and W. Maass, What Makes a Dynamical System Computationally Powerful?, in New Directions in Statistical Signal Processing: From System to Brains, edited by S. Haykin, J. C. Principe, T. J. Sejnowski, and J. G. McWhirter (MIT, Cambridge, MA, 2007), pp. 127-154.

[68] L. Büsing, B. Schrauwen, and R. Legenstein, Connectivity, Dynamics, and Memory in Reservoir Computing with 
Binary and Analog Neurons, Neural Comput. 22, 1272 (2010).

[69] J. Boedecker, O. Obst, J. T. Lizier, N. M. Mayer, and M. Asada, Information Processing in Echo State Networks at the Edge of Chaos, Theory Biosci. 131, 205 (2012).

[70] L. Livi, F. M. Bianchi, and C. Alippi, Determination of the Edge of Criticality in Echo State Networks through Fisher Information Maximization, IEEE Trans. Neural Networks Learning Systems 29, 706 (2017).

[71] P. C. Hohenberg and B.I. Halperin, Theory of Dynamic Critical Phenomena, Rev. Mod. Phys. 49, 435 (1977).

[72] M. Prokopenko, J. T. Lizier, O. Obst, and X. R. Wang, Relating Fisher Information to Order Parameters, Phys. Rev. E 84, 041116 (2011).

[73] L. Mazzucato, A. Fontanini, and G. La Camera, Stimuli Reduce the Dimensionality of Cortical Activity, Front. Systems Neurosci. 10, 11 (2016).

[74] J. A. Hertz, Y. Roudi, and P. Sollich, Path Integral Methods for the Dynamics of Stochastic and Disordered Systems, J. Phys. A 50, 033001 (2017).

[75] J. Eccles, P. Fatt, and K. Koketsu, Cholinergic and Inhibitory Synapses in a Pathway from Motor-Axon Collaterals to Motoneurones, J. Physiol. (Lond.) 126, 524 (1954).
[76] S. Song, P. Sjöström, M. Reigl, S. Nelson, and D. Chklovskii, Highly Nonrandom Features of Synaptic Connectivity in Local Cortical Circuits, PLoS Biol. 3, e68 (2005).

[77] D. Marti, N. Brunel, and S. Ostojic, Correlations between Synapses in Pairs of Neurons Slow Down Dynamics in Randomly Connected Neural Networks, Phys. Rev. E 97, 062314 (2018).

[78] F. A. Rodrigues, T. K. D. Peron, P. Ji, and J. Kurths, The Kuramoto Model in Complex Networks, Phys. Rep. 610, 1 (2016).

[79] T. Nishikawa and A. E. Motter, Comparative Analysis of Existing Models for Power-Grid Synchronization, New J. Phys. 17, 015012 (2015).

[80] S. Allesina and S. Tang, The Stability-Complexity Relationship at Age 40: A Random Matrix Perspective, Population Ecol. 57, 63 (2015).

[81] A. Pomerance, E. Ott, M. Girvan, and W. Losert, The Effect of Network Topology on the Stability of Discrete State Models of Genetic Control, Proc. Natl. Acad. Sci. U.S.A. 106, 8209 (2009).

[82] J. W. Negele and H. Orland, Quantum Many-Particle Systems (Westview Press, Boulder, 1998).

[83] M. Abramowitz and I. A. Stegun, Handbook of Mathematical Functions: With Formulas, Graphs, and Mathematical Tables (Dover Publications, New York, 1974). 